\title{
Lactobacillus on Recovering of Immune-Damage by Chemotherapy (Cancer Treatment)
}

Shousong Yue, Shandong Academy of Agricultural Sciences, Jinan, China yueshousong@163.com

Zhenzhong Zhang, Shandong Academy of Medical Sciences, Jinan, China 1345034285@qq.com

Fei Bian, Shandong Academy of Agricultural Sciences, Jinan, China bxf.9@163.com

Yan Zhang, Shandong Academy of Agricultural Sciences, Jinan, China yanzi820106@126.com

Gao Chen, Shandong Academy of Agricultural Sciences, Jinan, China chengao001@aliyun.com

*Jun Li, Shandong Academy of Medical Sciences, Jinan, China

ykylijun@126.com

*Jean-François Picimbon, Shandong Academy of Agricultural Sciences, Jinan, China;

Qilu University of Technology, Jinan, China

jfpicimbon@gmail.com

jfpicimbon@163.com

ORCID 0000-0002-9440-4772

"To whom correspondence should be addressed.

Short title Improving cancer treatment by natural $3 L$ bioproduct 


\begin{abstract}
We demonstrated the role of natural probiotics 3L, 3 Lactobacilli, in the establishment of a strong and sustainable beneficial healthy gut flora, after chemotherapy through experimental results through in vivo model. Using rat CTX model (immunosuppression induced by cyclophosphamide), we suggested some new adjuvant to chemotherapy as drugs + lactobacillus treament. Further, we proposed a new probiotic formulation (L. acidophilus + L. casei + L. plantarum) to be explored in the prevention of health condition loss by alteration of the general immune system, in numerous studies that reported the use of probiotics involving Lactobacillus in the post-chemo or post-surgical procedures. Here, in our study, Illumina MiSeq sequencing was used to generate sequencing data from microbial genomic DNA libraries, which is appreciable to check for the effects of $3 \mathrm{~L}$ on bacteria. Microbiome analysis, phylogenetic and classification reports, community data have supported the experiments and the results where $3 \mathrm{~L}$ had strong beneficial effects on the microbiome. Further, the influence on specific metabolic pathways are assisted in deriving the conclusion of the study (use of 3L for cancer therapy) to the mode of action, mechanistically by correcting microbiota composition and enhancing specific gut metabolic functions.
\end{abstract}

Keywords: Illumina MiSeq sequencing - Gut microflora - Lactobacilli - Immunostimulant - Anticancer 


\section{Introduction}

Various research challenges are crucial for the cancer care continuum and to find remedy at different levels of the most threatening disease of human health [1]. Following an accumulation of mutational, genetic and epigenetic alterations, abnormal cells start to divide without control and soon form a mass of extra-tissue (or tumor) that can spread to the whole body through bloodstream and lymph and becomes deadly [2-5]. Diseasespecific mutational events can serve as reliable cancer biomarkers for diagnosis [6-7], which is particularly relevant since multiple forms of primary cancerous tumors can develop in human cells [8-10]. However, high diversity of genetic mutations in mitochondrial DNA, non-coding RNA, microRNA, ubiquitin, RNA editing, spliceosome and/or RNA splicing and phenotype-specificity of drug sensitivity that largely depends on tumor-stroma interactions are reasons for the difficulties in cancer treatment [11-18]. The origin of cancer may even reside in an alteration of the mechanisms involved in RNA + peptide mutations that are required for multifunction in specific cell lines, including pluripotent stem cells, chemosensory cells or $\mathrm{T}$ cell lymphocytes. Cancer may arise from a dysregulation in protein variance and cell multipotency, a new molecular insight and potentially novel clinical application in disease treatment [19-22].

Accordingly, numerous studies have been undertaken to test the effects of nanomedicine, bioengineering of extracellular vesicles, gene therapy, immunotherapy, radiotherapy and thermal ablation on cancer treatment [23-24]. Immunotherapy, i.e. the stimulation of the capacity of T lymphocytes for antigen-directed toxicity, has gained a particular attention in clinical practices against cancer [25]. This is also strongly required not only because the immune system is targeted by the disease, but also because it is seriously affected by anticancer strategies such as (chemo)radiotherapy [26-28]. The side effects of ionizing radiation therapy, which is used by more than $50 \%$ in many categories of cancer cases, are late inflammatory responses and/or inflammation-associated diseases in the patient's immune system over a long period of time, especially in high dose rate radiotherapy [29-30]. One main concern is prompted by the fact that (chemo)radiotherapy can lead to a new type of cells (Langherans cells) known to reduce the immune system's ability to fight cancer [31]. Therefore, one of the key challenges for cancer research is to find new methods to keep using (chemo)radiotherapy that is strictly necessary to promote T-cell activity in order to cure the disease, while preserving the patient's natural barriers and immune functions to fight other infections such as pneumonitis 
[32-33]. The idea of turning to complementary and/or alternative more natural medicine to treat cancer is not new, but it may retain a more sustained consideration in the purchase of our modern life [34]. Given the link between the gut and the immune system, gut microbes (or microflora) have become an important target to boost $\mathrm{T}$ cells and shape a better efficiency of cancer therapy [35-36]. Using different cancer model studies, it has been shown that a rich gut flora strongly influences the effectiveness of anticancer drugs such as cyclophosphamides (CTX) and immune checkpoint inhibitors (ICIs) [37-40]. Our present study compares with any of the marketed probiotic formulations such as L. rhamnosus that are known to inhibit the growth of cancer cells in a dose- and time-dependent manner [41]. It also compares to L. rhamnosus GG, as the most studied L model in cancer, to potentiate the gut microbiota and protect against the genesis of tumors [35, 42]. In this new and challenging research field, mainly focusing on colon cancer, an universal 'probiotic' approach is expected to prevent patient selection in view of the different treatments and individualized host responses to gut modulation [43]. Accordingly, for medicinal purposes, we have developed a specific 3L Lactobacillus bioproduct (a natural cocktail composed of L. acidophilus, L. casei and L. plantarum), which has been demonstrated to have a very beneficial effect not only on physiological but also on biochemical status of hyperlipidemic mice. In mice, "3L" has been shown not only to induce a beneficial gut flora, but also to significantly reduce cholesterolemia, LDL/HDL ratio, blood lipid concentration and weight gain [44-45]. Rodents are the best models to study human metabolic syndromes and obesity-associated pathologies [46]. They are also very reliable models to investigate human cancer immunology and immunotherapy [47]. Therefore, we used five groups of rats as experimental models to check for the effects of 3L on gut flora and cancer chemotherapy. We analyzed the gut microbiome after chemotherapy (use of cytotoxic drugs such as CTX) for treatment of cancer by using Illumina MiSeq sequencing optimized for full complete microbial genome applications. We showed a microbiome composition significantly altered by chemotherapy. Then, we tested the effects of chemotherapy combined with $3 \mathrm{~L}$ on gut flora and health status of rats treated for cancer. The overall result of cancer treatment with CTX + Lactobacillus was beneficial to an extent not reported before. Increasing the dose of a Lactobacillus cocktail (acidophilus - casei - plantarum) had a marked positive effect on gut flora. Applying a microecosystem such as Lactobacillus did not cure cancer, but contributed to a better gut health in the chemotherapy for tumor treatment. A pharmacological bioproduct high in Lactobacillus strains is shown to significantly contribute to a healthy gut microbiome despite CTX 
chemotherapy. We therefore propose a new natural medicine to be applied in cancer treatment, in particular in prevention of an altered gut due to cyclophosphamide therapy.

\section{Materials and Methods}

Industrial preparation of Lactobacillus

In a pioneering study, we developped a three-strain lactobacillus probiotic formula (3L) against cholesterolemia and hyperlipidemia [43]. The same probiotic formula used in this study against immune damages in chemotherapy for cancer (L. acidophilus SD65, L. casei SD07 and L. plantarum SD02) was produced by our Industrial Laboratory platform for natural medicine in Jinan (Shandong Province, P.R. China). The growth of pure cultures of the three bacterial strains was taken in de Man, Rogosa and Sharpe (MRS) agar liquid medium and placed in an anaerobic workstation held at $37^{\circ} \mathrm{C}$ (industrial platform), following Yue et al. (2014) [43]. For 3L preparation, the bacterial cells of each strain were harvested by centrifugation at $2000 \times \mathrm{g}$ for $20 \mathrm{~min}\left(4^{\circ} \mathrm{C}\right)$. The cell pellet in each strain was resuspended in the ratio of $10^{9} \mathrm{CFU} / \mathrm{ml}$ in sterile saline water solution and kept at $4^{\circ} \mathrm{C}$. The tripartite $\mathrm{L}$ probiotic solution $(3 \mathrm{~L})$ was freshly prepared by mixing the cold suspensions of SD65, SD07 and SD02 in an equal volume and stored in cold conditions $\left(4 \sim 10^{\circ} \mathrm{C}\right)$ and used on later point of time. Accordingly, rats received every day a dose $(0.3$ $\mathrm{ml}$ ) of bioproducts administered intra-gastrically using a stainless-steel needle in addition of chemotherapy (cyclophosphamide, CTX; Figure 1).

Preparation of five groups in rat CTX model (immunosuppression induced by CTX)

Rats were purchased from Jinan Pengyue Laboratory Animal Company (Product license SCXK (LU) 20140007). Fifty healthy Specific Pathogen Free (SPF) Sprague-Dawley (SD) male rats (N = 50 males, young adults, 8-9-week-old; body weight: 260-316 g) were divided into five experimental groups: 1) Control healthy (CK), 2) Immune attacked (IM), 3) Immune attacked and treated with low dosage of Lactobacillus, i.e. $1.25 \mathrm{ml} / \mathrm{kg}$ bodyweight (L), 4) Immune attacked and treated with middle dosage of Lactobacillus, i.e. 2.5 
$\mathrm{ml} / \mathrm{kg}$ bodyweight (M), and 5) Immune attacked and treated with Lactobacillus, i.e. $5.0 \mathrm{ml} / \mathrm{kg}$ bodyweight (H). Total number of studied animals was 50, in each group were 10 rats (Figure 1). Control rats (CK group) were with continuous gastric perfusion of normal saline (NS). Rats in groups 2-5 were immune attacked by an injection of cyclophosphamide (CTX, $10 \mathrm{mg} / \mathrm{ml}$, intraperitoneally). "Nude" rats were only treated with CTX at day 1, 5, 8, 15 and 22 (IM group; Figure 1). "Covered" rats were with continuous gastric perfusion of NS containing a specific dosage of $3 \mathrm{~L}(1.25 \mathrm{ml} / \mathrm{kg}$, low dose, L group; $2.50 \mathrm{ml} / \mathrm{kg}$, middle dose, M group; $5.0 \mathrm{ml} / \mathrm{kg}$, high dose, H group; Figure 1). The deleterious effects of CTX cyclophosphamide chemotherapy on the rat immune system and the beneficial effects of Lactobacillus preparation on immuno suppression induced by cyclosphosphamide were highlighted in a companion study [48]. We found that from one week to one month (28 days) after administration, the number of white blood cells of the animals treated with Lactobacillus preparation (H-dose: $5 \mathrm{mg} / \mathrm{kg}$ ) was higher than that of the animals in the model group treated with CTX. The CD4+/CD8+ ratio (ratio of T helper cells to cytotoxic T cells) was also higher in the animals treated by Lactobacillus preparation (L-dose: 1.25 , M-dose: 2.5 and H-dose: $5 \mathrm{mg} / \mathrm{kg}$ ). Lactobacillus-treated animals showed increased serum level of interleukin 6 (IL-6) and high interleukin (IL-6 and IL-2) gene expression, but significant decrease in the rat mRNA expression levels of Tumour Necrosis Factor alpha (TNF-alpha). The effects on leukocytes, T helper cells, interleukins and TNF-alpha were dose-dependent [48], which urged us to test three rather different doses (H, M and L) on the microbiome of rat models for chemotherapy. Following the results from Zhang et al. (2020) on chemotherapy and immuno-depression [48], the follow up of the present experiment on chemotherapy and microbiome was about 28 days (D28). Fecal samples were collected only once on D28 in each rat group after about one month treatment (five CTX injections chemotherapy). Rat feces corresponding to $\mathrm{CK}, \mathrm{IM}, \mathrm{L}, \mathrm{M}$ and $\mathrm{H}$ groups were stored at $-80^{\circ} \mathrm{C}$ until DNA extraction, Illumina MiSeq Sequencing and comparison of microbiome profilings between the five groups (Figure 1). 


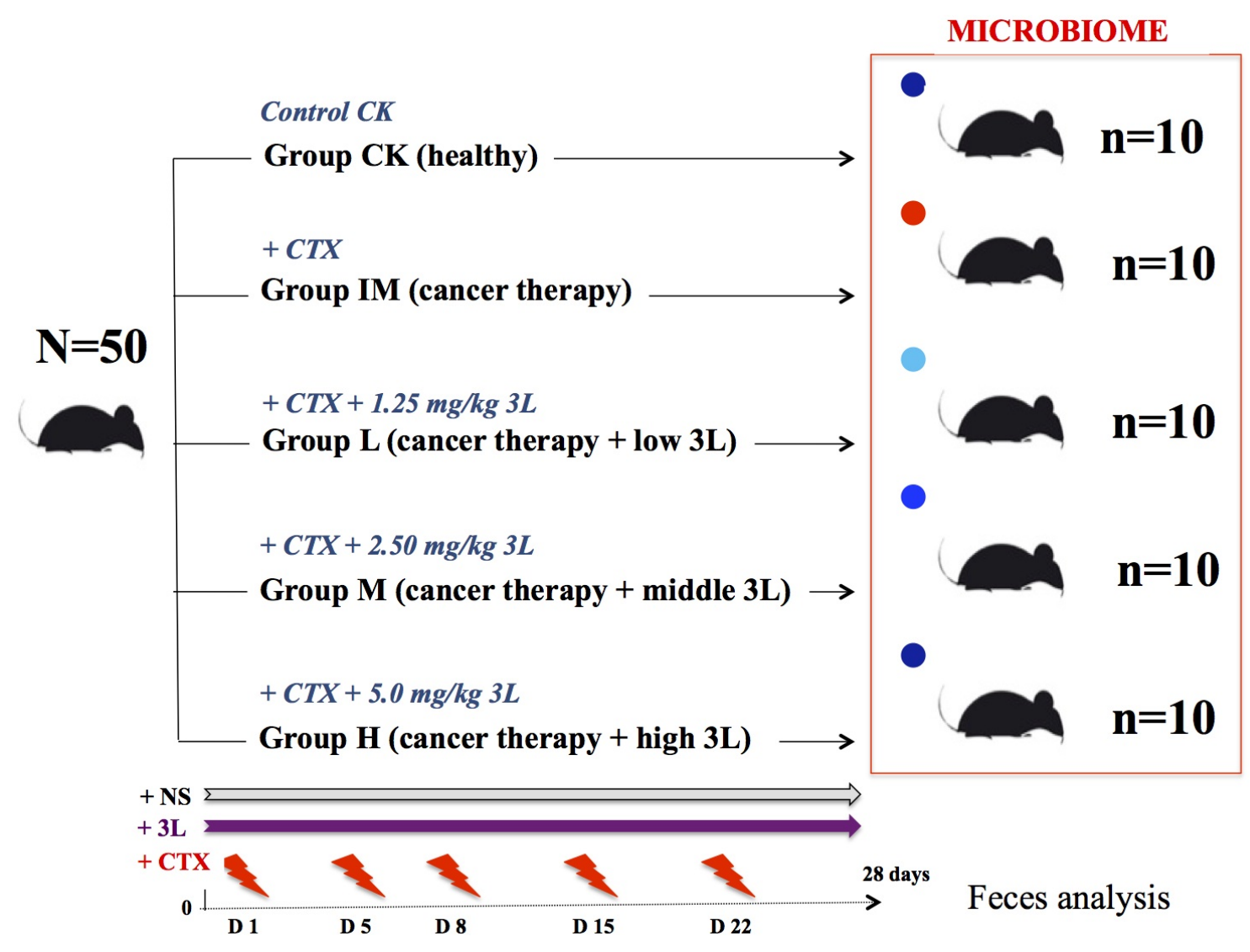

Figure 1 Experimental model of testing Lactobacillus tritherapy on rats in cancer chemotherapy conditions.

The red dot shows the immune-attacked group with depleted gut flora after twenty-eight days of chemotherapy (cyclophosphamide CTX, $10 \mathrm{mg} / \mathrm{ml}$ ). Rats received an injection of CTX on D1, D5, D8, D15 and D22, respectively. Normal saline (NS) was delivered by continous perfusion. In groups L, M and H, rats received a daily medication (one dose) of probiotic Lactobacillus (3L: L. acidophilus SD65, L. casei SD07 and L. plantarum SD02). L: low dose (1.25 mg/kg), M: middle dose (2.50 mg/kg), H: high dose (5.0 mg/kg). The blue dots show the groups that have maintained gut flora close to control healthy conditions (Lactobacillus-treated groups). The dark blue dot shows the group (high $3 \mathrm{~L}$ ) that has gut flora (microbiome) highly similar to control healthy conditions (CK). Time 0 is when chemotherapy starts in + CTX groups. Feces were collected in each group after twenty-eight days treatment. $\mathrm{N}=$ total number of rats in experiment, $\mathrm{n}=$ number of rats per group. 
Preparation of five fecal microbial genomic DNA samples in rat CTX model

For the preparation of DNA samples from the five groups of rats (CK, IM, L, M and H) in rat CTX model, DNA was extracted using the method previously selected for mice and piglet fecal microbiome analysis [43$44,49]$. This method was reliable for testing, quantity, purity and quality control of fecal DNA samples and Illumina sequencing [49]. As described in Yue et al. (2020) [49], $2.0 \mathrm{~g}$ of fecal samples from Group CK-H rats were processed for microbial genomic DNA extraction using QIAamp Fast DNA Stool MiniKit (Qiagen GmbH, Hilden, Germany) and used as template (10 ng) in PCR reactions employing 338F and 806R universal primers: 338F 5'-ACTCCTCGGGAGGCAGCA-3' and 806R 5'-GGACTACHVGGGTWTCTAAT -3' (Personal Biotechnology Co, Ltd, Shanghai, China) for 16S samples (V3-V4, 480 bp, Miseq-PE250). For ITS (ITS1, 250 bp, Miseq-PE250), PCR reactions employed ITS5F 5'-GGAAGTAAAAGTCGTAACAAGG-3' and ITS1R 5'-GCTGCGTTCTTCATCGATGC-3' (Personal Biotechnology Co, Ltd, Shanghai, China). Each sample for Illumina sequencing corresponded to three individuals from the same group. Accordingly, fifteen different samples (CK: C101, C103, C105; IM: IM015, IM021, IM024; L: L102, L103, L104; M: M201, M202, M203; H: H105, H204, H205) were subject to Illumina MiSeq (NCBI SubmissionID: SUB9725559; BioProject ID: PRJNA754332; BioSamples: SAMN20769197-SAMN20769206; Accession Numbers: SRX11740945-SRX11740959). During microbiome analysis in relation with cancer and chemotherapy, three biological samples were tested in each group of rats, so we conducted individuals, replicates and comparison groups.

Before sequencing, PCR amplification of $16 \mathrm{~S}$ rDNA products (TransGen Biotech, Beijing, China) was carried out in a Takara Master Thermal Cycler Dice (Takara, Dalian, China) programmed for an initial denaturation of $95^{\circ} \mathrm{C}$ for $3 \mathrm{~min}$ followed by 30 cycles of $\left(94^{\circ} \mathrm{C}\right.$ for $\left.30 \mathrm{~s}\right), 50^{\circ} \mathrm{C}$ for $30 \mathrm{~s}, 72^{\circ} \mathrm{C}$ for $1 \mathrm{~min}$ and a final extension of $72^{\circ} \mathrm{C}$ for $7 \mathrm{~min}$. PCR amplification used Q5® high-fidelity DNA polymerase (New England BioLabs Inc., Ipswich, Massachussets, USA). PCR products or amplicons for each group were purified by $2 \%$ agarose gel electrophoresis (Bio-Rad Beijing, China) and using gel recovery kit (Axygen ${ }^{\circledR}$, AxyPrep DNA gel extraction kit, New York, USA). The PCR product concentration was determined using a fluorescence reagent-based method (Quant-iT PicoGreen dsDNA Assay Kit, Fisher Scientific ${ }^{\mathrm{TM}}$, Loughborough, UK) in microplate reader (BioTek $\left.{ }^{\mathrm{TM}}, \mathrm{FLx} 800^{\mathrm{TM}}\right)$. 


\section{Illumina MiSeq Sequencing}

Illumina MiSeq sequencing was used to generate sequencing data from microbial genomic DNA libraries in the five groups of rats (CK-H) for alternative therapy and cancer research. For Illumina MiSeq, we used Illumina's TruSeq Nano DNA LT Library Prep Kit as for human genome and gut microbiota sequencing (Human Genome Assembly: The Genome Sequencing Consortium, 2001) [50]. The purpose in MiSeq was to add adapter sequences onto the ends of microbial DNA fragments to generate indexed libraries for both single- and paired-end reads [51]. Firstly, fecal microbial genomic DNA amplicons from rats were subject to terminal end repair. The 5'-end of DNA was excised by End Repair module (Mix2), and added with a phosphate group. Meanwhile, the missing base of 3'-end was filled. Adenosine base was added at the 3'-end of each microbial DNA sequence for prevention of self ligation. This also insured that each DNA target sequence was properly linked to the sequencing linker. A sequencer corresponding to a library-specific tag (Index Sequence) was added at the 5'-end of the PCR amplicons to immobilize DNA in flow cell. Self-ligated fragments were removed using BECKMAN AMPure XP Beads (Beckman Coulter ${ }^{\mathrm{TM}}$, Illkirch, France) to purify the microbial library system. To enrich the libraries as much as possible for the DNA of interest, PCR amplicons were used as a template in a second-PCR run. The PCR conditions were as described under preparation of five fecal microbial genomic DNA samples in rate cancer model. In MiSeq, PCR amplicons were purified using the magnetic beads screening method (Beckman) and analyzed by $2 \%$ agarose gel electrophoresis before high-throughput sequencing.

Prior to high-throughput sequencing, the quality of each rat fecal microbial genomic DNA library was checked by using Agilent High Sensitivity DNA kit on Agilent Bioanalyzer (Agilent Technologies Inc. , Waldbronn, Germany). Each DNA library produced only a single peak and no joints on Agilent check. Sequence librairies were then quantified on the Promega Quantifluor fluorescence quantitative system using Quant-iT PicoGreen dsDNA assay kit (Promega Corporation, Madison, USA). Library concentration was above $2 \mathrm{nM}$. After serial dilutions, DNA samples were mixed, denatured by $\mathrm{NaOH}$ and sequenced. Two-end sequencing with $2 \times 300$ bp reading length was performed using Illumina MiSeq Reagent kit v3 on 600 cycles MiSeq Sequencer (Illumina Next Generation). The optimal sequencing length of the target DNA fragment was comprised between 200 and $450 \mathrm{bp}$. 
Reading sequences were identified and assigned to the corresponding samples based on primers and barcode information. All chimeric sequences (or artifacts formed by sequences incorrectly joined together) were removed using USEARCH (v5.2.236, http://www.drive5.com/usearch) [52]. Unreliable sequences (replication errors, nucleotide base substitutions, insert deletions, etc) for microbial genomic DNA were identified using QIIME (Quantitative Insights Into Microbial Ecology, v1.8.0, http://qiime.org) [53]. Sequences with more than one base mismatched with the 5 '-end primer and/or more than eight consecutive identical bases were discarded [52-54]. Other sequences were classified into Operational Taxonomic Units (OTUs), which were further used for taxonomic identification of bacterial DNA and phylogenetic analysis [55]. The diversity level of each sample was evaluated on the basis of OTU values, while the depth of sequencing (enhanced microbial community analysis) was shown by analysis of rarefaction curves [56]. The composition of the five samples corresponding to the five groups of rats (CK-H) was analyzed at different taxonomic levels: phylum, order, class, family, genus and species (i.e. complete microbiome; Figure 1). The microbiome results (OTU, 100\%) were displayed in tables and histograms (R software). Rare OTUs (with an abundance proportion less than $0.001 \%$ ) were not considered for microbiome analysis [57]. Shared and unique OTUs within each group (calculated by R) were displayed in Venn diagrams in R (Treat*/2.5.1_Venn). Using OTU abundance matrix to study complete microbial structure in each group, rarefaction curves were drawn to reflect the microbial diversity among samples, i.e. compare the number of OTUs in the five different groups of rats (CK-H) at the same sequencing depth and justify the diversity level of each sample (QIIME2, alpha rarefaction curve). The length of the curve reflects the number of sample sequencing depth; the longer the curve, the higher the sequencing depth, which strongly increases the possibility to observe increased microbial diversity. The slope of the curve reflects the effect of sequencing depth on microbial diversity in the sample. A flat rarefaction curve (low slope) indicates that the sequencing results are sufficient to reflect microbial diversity, and increasing sequencing depth cannot detect more novel OTUs. A bump rarefaction curve (high slope) indicates that the diversity is not saturated, increasing sequencing depth could help detect more OTUs (Treat*/2.3.2_arare). We also measured "Specaccum" (species accumulation curve) in five groups of rat associated with cancer treatment and 3L. Similar to rarefaction curve, specaccum gives the extend of increase in microbial community richness along with increase in sample size [58]. Using specaccum function in R, we estimated whether the sample size was sufficient to reflect the different underlying bacterial 
communities of the different groups or samples. The specaccum species accumulation curve was plotted for the total number of OTUs in each sample from the OTU abundance matrix using R in vegan (S3 method; Treat*/2.3.3_specaccum) [59]. In addition, rank abundance curve (RAC) was used to see the number of highly abundant vs rare OTUs in each community [60]. For RAC, OTU values were in descending orders and transformed in Log2 data in R package (Treat*/2.3.4_rabund). Other multiple indices were scored to reflect microbial alpha diversity in rat groups. We included Chao1 index and ACE index to reflect community richness, and Shannon-Simpson indices to reflect both evenness and richness of the bacterial community in each of the five groups of rats related to cancer chemotherapy and lactobacillus treatment (CK-H) using QIIME software (QIIME 2) in R [54-60].

The differences in gut flora structure and related microbial species between the groups were analyzed by multiple statistical analysis tools (Metastats) in Mothur software (http://metastats.cbcb.umd.edu), providing the sequence difference (or absolute abundance) of two samples/groups on the basis of P and Q values [61]. LEfSe was used to measure community composition differences in the different groups based on linear discriminant analysis (LDA) effect size. LEfSe analysis combines LDA with Krustal-Wallis and Wilcoxon rank sum test to find key biomarkers (i.e, key community members) [62]. Relative abundance matrix was used for LEfSe analysis through the Galaxy Online Analysis Platform for sample group comparison and visual analysis results (http://huttenhower.sph.harvard.edu/galaxy; Treat*/2.5.5_LEfSe).

\section{Phylogenetic and classification analysis}

OTU representative sequences were used as taxa to build phylogenetic trees (Newick) in FastTree tool [63]. Using MEGAN [64], abundance and taxonomic composition information of OTUs in each sample was projected to the microbiological classification tree from NCBI (https://www.ncbi.nlm.nih.gov/taxonomy). Hierarchical trees (GraPhlAn) were constructed with the whole sample population at each taxonomic level. Using GraPhlan [65], taxonomic units were distinguished by different colors and their abundance distribution was reflected by the node size, i.e. the average relative abundance of the taxonomic unit. Interactive presentations of community taxonomy in groups of rats related to cancer were conducted by KronaTools 2.4 
software [66]. The main purpose of $\beta$-diversity analysis was to examine the similarity of community structure between the different groups. Three different types of methods were used to observe the differences between the groups through the natural decomposition of the community data structure and the sample ordination: principal component analysis (PCA), multidimensional scaling (MDS) and clustering analysis (CA). PCA evaluates the similarity between samples based on Euclidean distance, regardless of the possible inter-relation between the original variables ( $\mathrm{R}$ software). Nonmetric MDS only considers the size of the relationship between samples before to classify the group or sample distances (UniFrac distance matrices of Unweighted and Weighted in R software). Like Nonmetric MDS analysis, CA such as Unweighted Pair-Group Method with Arithmetic Means (UPGMA), single-linkage clustering and complete-linkage clustering uses any distance to evaluate the similary between samples (QIIME-R). Using QIIME, the Weighted and Unweighted UniFrac distance between or within the groups were tested by T-test. The statistical significance was checked by 1000 Monte Carlo permutations. This fully describes the size of the flora structure differences between or within groups of rat fecal samples related to cancer.

Finally, the massive community data produced by high-throughput DNA sequencing in five groups of rats (CK-H) required to use more statistical analyses such as Constrained Ordination and Supervised Learning. In accordance with a known sample correlation (a sample distribution or grouping information) or a sample test indicator (continuous variable), the commonly used Constrained Ordering and Supervized Learning methods, including redundancy analysis (RDA), canonical analysis (CNA) and partial least squares discriminant analysis (PLS-DA, Variable Importance in Projection or VIP value in R) extracted the pattern characteristics associated with original microbial community data. The larger the VIP value is, the greater is contribution of the species to the differences between groups. In addition, Adonis/PERMANOVA (permutational multivariate analysis of variance) analysis was performed using QIIME software, and 999 substitutions were used to determine whether the differences between groups were statistically different. Focusing on $P$ value, the smaller the $P$ value, the stronger the difference between groups of rats for cancer. 


\section{Results}

\section{Comparison of microbiomes in rat groups in relation with chemotherapy and Lactobacillus}

In the continuity of our work on Lactobacillus/bacillus on cholesterolemia, lipidemia, diarrhea and scour [see 43-44, 49], this new study explores the fecal microbiota of rats subject to chemotherapy (cyclophosphamides) for cancer and significant health improvement when co-treated with a cocktail of three Lactobacillus spp. The topic is of very high significance in pharmacological treatments of cancer, as cyclophosphamides or CTX used against cancerous tumors are commonly found to severely damage the patient's immune system. The bioproduct for cancer study design includes five different experimental groups (1) healthy control rats and treated rats (CTX chemotherapy), which were either only treated with CTX (2) or treated with CTX and a low (3), middle (4) or high (5) complement dose of Lactobacillus spp (Figure 1). In each group (1-5), leukocyte concentration, CD4/CD8, interleukin and TNF-alpha expression were measured [see 48]. Here, in each group (1-5), the microbiome was assessed using 16S rRNA gene sequencing using Illumina MiSeq platform (Figures 2-5 \& S1-S13 and Tables 1-2 \& S1-S4).

The pictorial representation of the relationships between the five groups (Venn Diagram) in relation with chemotherapy and Lactobacillus showed a strong relation between healthy control and CTX + high dose of Lactobacillus spp. (CK and H; Figure 2). Importantly, the position, the configuration and overlap of the circles indicating the relationships between the groups showed a gradual increase in gut flora and general healthy conditions depending on the complement dose of Lactobacillus spp. (L-to-H; Figure 2). The Venn Diagram was such that the core of microbiomes in the $\mathrm{H}$ and controls groups was closely related, while low complement dose of Lactobacillus spp. remained too closely related to CTX alone conditions (Figure 2). Middle complement dose $(\times 2$, two-times fold) fell in between control (CK) and immune-damaged (IM) groups (Figure 2). Therefore, increasing Lactobacillus spp. concentration (no more than $\times 5$, five-times fold) was necessary to provoke a strong beneficial effect on the microbiome of rats treated by chemotherapy for cancer (Figure 2). 


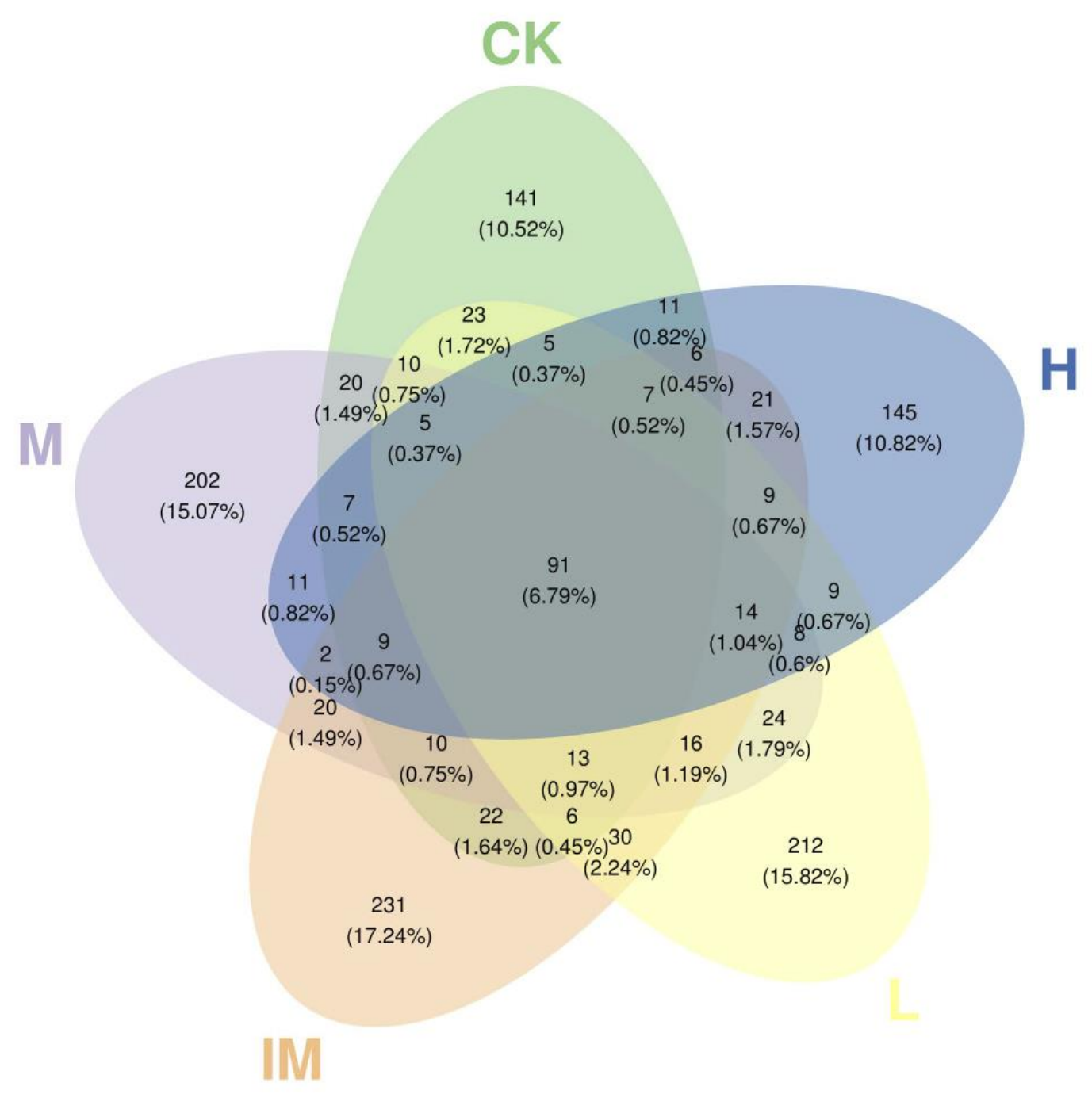

Figure 2 Venn diagram of shared OTUs across different rat groups in relation with cancer and chemotherapy. Different colors represent different groups: 1) normal (control healthy, CK), 2) ill (immune-damaged, IM), 3) chemotherapy +3L-low dose (L), 4) chemotherapy + 3L-middle dose (M) and 5) chemotherapy + 3L-high dose $(\mathrm{H})$. The interior of each circle symbolically represents the number and percentage of shared microbes in each sample or group. The overlapping area or intersection of circles represents the set of OTU microbes commonly present in the counterpart samples or groups (CK, H, M, L, IM). The single-layer zone represents the number of OTUs uniquely found in specific rat sample/group (R(v3.1.1), BGI Co., Ltd). 
All samples (three samples per group) and OTU numbers were coherent between the groups CK-H. About the same quantity of $16 \mathrm{~S}$ and ITS sequences was obtained in different biological replicates in each group (Table S1). About the same sequence quantity was obtained in five different groups for chemo (Table S1). No differences were found in the OTU number of samples at each taxonomic level (phylum, class, order, family, genus and species; Tables S2 \& S3). All of the OTUs could be classified, but the classification of OTUs at different taxonomic levels in five rat groups related to chemotherapy and Lactobacillus treatment did not show apparent differences in the OTU counting (Tables S2 \& S3). This was observed for grouped (CK, IM, L, M and H) and ungrouped (C101, C103, C105, IM015, IM021, IM024, L102, L103, L104, M201, M202, M203, H105, H204 and H205) samples (Tables S2 \& S3). Therefore, the sample sizes were the same, especially for Family and Genus (Figure S1). However, using the OTU table for sample diversity in PCA, rank abundance curve, NMDS, principle coordinate analysis (PcoA), Bray-Curtis distance plot (default semimetric), binary Jaccard distance matrix (metric) and UPGMA clearly classified H with CK.

Not only Venn Diagram, but also PCA based on the OTU composition displayed key differences in the five groups (Figure S2). PCA showed similarity of the chemotherapy + high lactobacillus and control groups at overall community level, with Fusarium, Talaromyces, Sarocladium, Aspergillus and Mucor falling outside a common spectrum of microbe genera (Figure S2A).

Principle Component Analysis and Orthogonal Projections to Latent Structures Discriminant Analysis (OPLS-DA) showed Mucor and Talaromyces associated to ill conditions (chemotherapy alone), but a high diversity of microbes associated to control and chemotherapy+high Lactobacillus conditions (Figure S2B). OPLS-DA analysis provided clear evidence that distances between CK and $\mathrm{H}$ groups were very small, while L, M and IM clearly separated. The overlapping distributions of the two groups, $\mathrm{CK}$ and H, corresponded to specific fungi, including Fusarium, Sarocladium, Kodamaea, Verticillium, Candida and Chlamydomyces. Trichoderma, Acremonium and Malassezia were more associated to H, M and L Lactobacillus groups. Pichia and Aspergillus separated from this group, but combined with CK control. Like Mucor and Talaromyces, Xeromyces, Xerochrysium and Penicillium microbial fungi fell with CTX and immune-damage (Figure S2AB). 
Similarly, OTU rank abundance curve (i.e. species richness and species evenness) showed more microbial diversity occurring in control and Lactobacillus (L-H) groups compared to CTX alone immune damaged ill group (Figure S3). In NMDS, CK-samples tended to group with H-, M- and L-triplicates. IM group fell distantly from the other groups, clearly showing mean dissimilarities between microbiomes from CTX-treated samples and those from controls (no treatment) and rats treated with Lactobacillus in addition of CTX (Bray-Curtis; Figure S4A). The NMDS graph using Jaccard index collapsed very clear information: CK grouped with $\mathrm{H}$-samples, showing mean similarities between $\mathrm{H}$-microbiomes and controls (Jaccard; Figure S4B). The same grouping was observed using PcoA as a principle analysis (Figure S5). Both BrayCurtis (abundance) and Jaccard (0/1 data) indices showed the pair of communities with similar species richness (H and CK; Figure S5AB). Unweighted and non-metric MDS analysis (UPGMA) confirmed the similarity between $\mathrm{CK}$ and $\mathrm{H}$ samples. On the UPGMA tree, $\mathrm{H}$ branches clustered with $\mathrm{CK}$ with low distance value (0.005-0.0028; Figure S6A). Unweighted pairs were also found between M and L groups (0.031-0.111, Figure S6A; 0.003, Figure S6B). The branches corresponding to the immune-damaged group (IM) clustered at the bottom of the tree, showing mean distance (or difference) of IM compared to CK, H, M and L (Figure S6).

Profiling microbiome in five different groups of rats in relation with cancer chemotherapy treatment and addition of Lactobacillus pharmacology. Specaccum (number of species vs number of samples) gave the extend of increase in microbial community richness along with increase in sample size. Specaccum estimated that the sample size was sufficient to reflect the different underlying microbial communities of the different rat groups (Figure S7). Chao1, Simpson, Shannon, Pielou_e, observed species and Goods_coverage indices (alpha-diversity) were scored in grouped and ungrouped samples (Figure S8 \& Table S4). These indices (Chao1, Pielou_e and observed_species) reflected similar community richness and species evenness in CK and $\mathrm{H}$ groups (Figure S8 \& Table S4). Goods_coverage index showed marked differences between H and IM. High microbial diversity was found in $\mathrm{H}$, as reflected by Goods_coverage metrics at OTU levels (sample completeness, $\mathrm{p}=0.76$; Figure S8 \& Table S4). Shannon-Simpson indices reflected high evenness and richness of the microbial community in each of the three Lactobacillus groups of rats related to cancer chemotherapy and probiotic treatment (Figure S8 \& Table S4). 
In the next step of the study of Lactobacillus for cancer therapy, microbial taxa clustering based on the abundance of each microbe in five rat samples related to cancer and treatment was analyzed by heatmap on group and triplicate samples (Figure 3AB). Microbe heatmap analysis showed the relative abundance of each taxon in CK, IM, H, M and L. The diverse microbial taxa identified in the rat groups for cancer and $3 \mathrm{~L}$ are listed in Table 1. There were several broad types of microbes identified in CK group from Acaulium (syn. Scopulariopsis) genus to Xeromyces (Figure 3 \& Table 1). CK (control, healthy condition) was characterized by high levels of Candida, Cutaneotrichosporon, Filobasidum, Fusarium, Kernia, Kodamaea, Lecanicillium, Meyerozima, Papiliotrema (Cryptococcus), Pichia, Rhodoturula, Verticillium and Wallemia, while IM (immune-attacked) showed high abundance of Acaulium, Mucor, Olpidium, Penicillium, Periconia, Phallus, Xerochrysium and Xeromyces (Figure 3 \& Table 1). Interestingly, however, treating rats with Lactobacillus in addition of Cyclophosphamide increased relative fecal abundance of many various microbial genera, including Acremonium, Aspergillus, Chlamydomyces, Fusarium, Mortierella, Phallus, Rasamsonia, Rhizophlyctis, Rhizopus, Talaromyces and Trichoderma (in H group), Coprinellus, Microascus, Mycosphaerella, Phialocephala, Pseudogymnoascus, Rhizomucor, Rhizophlyctis, Rhizopus, Sarocladium, Scytalidium, Thermoascus and Ustilago (in M group), and Mallassezia, Plectosphaerella, Rhizophlyctis, Rhizopus, Saccharomyces, Simplicillium, Sodiomyces and Tausonia (in L group; Figure 3 \& Table 1). Therefore, among regulated fungi are species which are not known as commensals or pathogens of animals. Lecanicillium are known as generalist entomopathogenic fungi [67]. Ustilago is known pathogen of Poaceae plants [68]. Phallus are big saprotrophic mushrooms [69]. The presence of these fungi in the rat microbiota is not necessarily doubtful and deserves attention. The breeding history of rats takes place in Class II animal facility of the Institute of Medicine in SAMS (Specific Free Pathogen/SPF facilities and acute hospital care settings that are designed to maintain organisms in sterile environments). Saprophytic basidiomycetes are known wood-decaying fungi, but they are also reported from penis and urethra of animals, where they play a role in male fertility [70]. In fact, little is known about the fungal floral of the digestive and reproductory tracts in rodents. Species in Lecanicillium are pathogens that parasitize not only insects, but also worms and many other fungi, which may explain their presence in gut fungi associated with rats. Various strains of Lecanicillium are found in gut fungi associated with marmots [71]. As seen in rodents, a large variety of 'forgotten' odd fungi, including Ustilaginales and Ustilago sp. , are found in the human digestive tract [72- 
75]. So, it is not so doubtful, odd or surprising that fungal sequences such as Lecanicillium, Phallus and Ustilago are found in the fecal DNA from CTX-rat models. It has been found in many other animal species, including humans. What is more surprising is to find that these fungi are differentially regulated by CTX and/or 3L conditions, which is the most important key point we want to make here (Figure 3, Table 1).
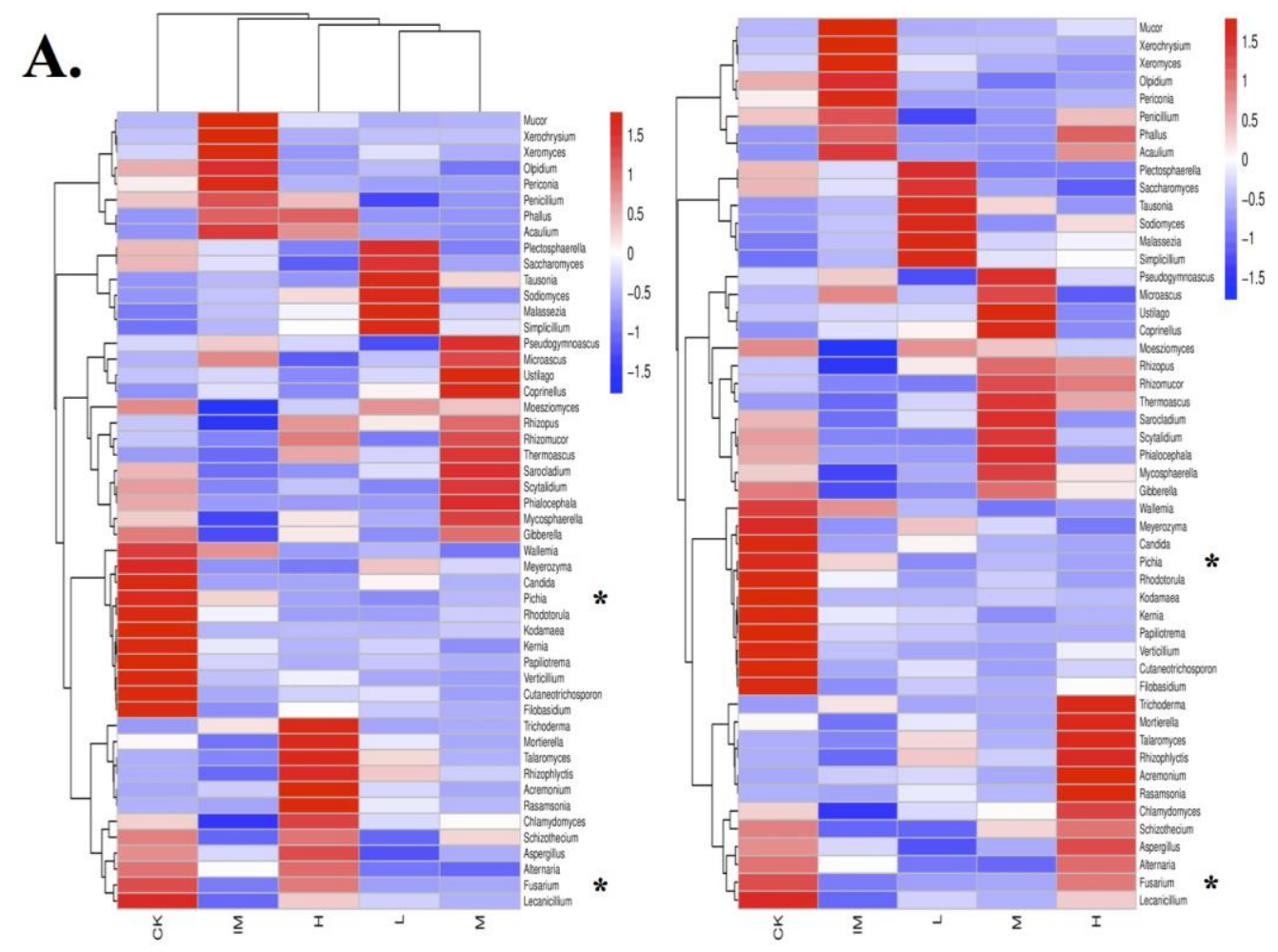

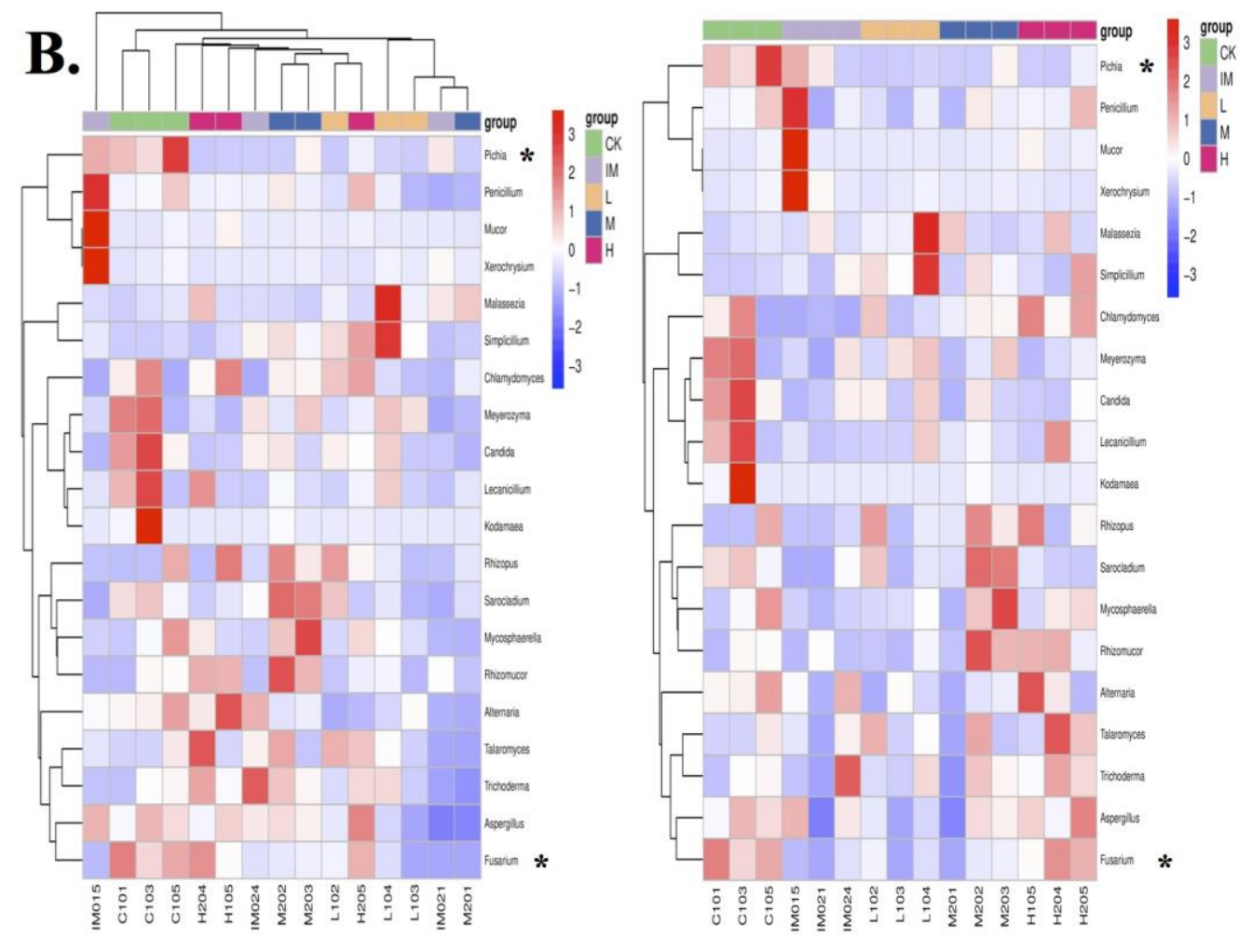

Figure 3 Fungal species clustering based on the abundance of each species (heat map) in rat samples in relation with cancer and chemotherapy. Log-scaled percentage heat map of fungal genus level in rat groups. Different colors represent different groups: 1) normal (control healthy, CK), 2) ill (immune-damaged, IM), 3) chemotherapy $+3 \mathrm{~L}$-low dose (L), 4) chemotherapy $+3 \mathrm{~L}$-middle dose (M) and 5) chemotherapy $+3 \mathrm{~L}$-high dose $(\mathrm{H})$. Longitudinal clustering indicates the similarity of all fungal genera among the five rat groups. The horizontal clustering indicates the similarity of certain fungal genera among the five rat groups $(\mathrm{CK}-\mathrm{H})$. The closer the distance is and the shorter the branch length is, the more similar the genus composition is between the groups and samples. A) Comparative analysis of CK-H groups. A total of fifty differentially regulated fungi are shown in the genus-heat map of rat groups in relation with anticancer cyclophosphamide drug, CTX-chemo and Lactobacillus. B) Comparative analysis of three different samples (or replicates) in each group (CK: C101, C103, C105; IM: IM015, IM021, IM024; L: L102, L103, L104; M: M201, M202, M203; H: H105, H204, H205). A total of twenty differentially regulated fungi are shown in the genus-heat map of triplicates in rat groups in relation with chemotherapy and 3L treatment. Left: Hierarchical clustering based on both groups and taxa (variables: columns and rows). The linkage between groups or samples is based on relative abundance of specific fungal genera (map both clustered). Right: Hierarchical clustering on taxa 
(variable: rows). The ordering method of groups or samples is independent of relative abundance of fungi (map taxa clustered). * shows differential levels of Pichia and Fusarium in CK/IM-L/M/H and CK/HIM/L/M groups, respectively. The amounts of individual fungal genera are represented as color gradients. Color gradation from red firebrick to navy-blue represents decreased amounts in fungal genus abundance (R(v3.1.1), BGI Co., Ltd). 


\begin{tabular}{|c|c|c|c|c|}
\hline $\mathrm{CK}$ & $\mathrm{IM}$ & $\mathrm{H}$ & $\mathrm{M}$ & $\mathrm{L}$ \\
\hline Acaulium & Acaulium & & & \\
\hline Acremonium & & Acremonium & & \\
\hline Alternaria & & & & \\
\hline Aspergillus & & Aspergillus & & \\
\hline Candida* & & & & \\
\hline Chlamydomyces & & Chlamydomyces* & & \\
\hline Coprinellus & & & Coprinellus & \\
\hline $\begin{array}{l}\text { Cutaneotrichosporon } \\
\text { Filobasidium }\end{array}$ & & & & \\
\hline Fusarium* & & Fusarium* & & \\
\hline Gibberella & & & & \\
\hline Kernia & & & & \\
\hline Kodamaea & & & & \\
\hline Lecanicillium & & & & \\
\hline Mallassezia & & & & Mallassezia \\
\hline Meyerozima & & & & \\
\hline Microascus & & & Microascus & \\
\hline Moesziomyces & & & & \\
\hline Mortierella & & Mortierella & & \\
\hline Mucor & Mucor & & & \\
\hline Mycosphaerella & & & Mycosphaerella & \\
\hline Olpidium & Olpidium & & & \\
\hline Papiliotrema & & & & \\
\hline Penicillium & Penicillium & & & \\
\hline Periconia & Periconia & & & \\
\hline Pichia* & & & & \\
\hline Phallus & Phallus & Phallus & & \\
\hline Phialocephala & & & Phialocephala & \\
\hline Plectosphaerella & & & & Plectosphaerella \\
\hline Pseudogymnoascus & & & Pseudogymnoascus & \\
\hline Rasamsonia & & Rasamsonia & & \\
\hline Rhizomucor & & & Rhizomucor & \\
\hline Rhizophlyctis & & Rhizophlyctis & & \\
\hline Rhizopus & & Rhizopus $^{\circ}$ & Rhizopus $^{\circ}$ & Rhizopus $^{\circ}$ \\
\hline Rhodotorula & & & & \\
\hline Saccharomyces & & & & Saccharomyces \\
\hline Sarocladium & & & Sarocladium & \\
\hline Scytalidium & & & Scytalidium & \\
\hline Shizothecium & & & & \\
\hline Simplicillium & & & & Simplicillium \\
\hline Sodiomyces & & & & Sodiomyces \\
\hline Talaromyces & & Talaromyces & & \\
\hline Tausonia & & & & Tausonia \\
\hline Thermoascus & & & Thermoascus & \\
\hline Trichoderma & & Trichoderma & & \\
\hline Ustilago & & & Ustilago & \\
\hline $\begin{array}{l}\text { Verticillium } \\
\text { Wallemia }\end{array}$ & & & & \\
\hline Xerochrysium & Xerochrysium & & & \\
\hline Xeromyces & Xeromyces & & & \\
\hline
\end{tabular}

Table 1. Composition of the mycobiome (Genus of fungi and yeasts) identified in five rat groups in relation with cancer, chemotherapy and Lactobacillus treatment. Lactobacillus treatment: tritherapy $(3 \mathrm{~L})=$ L. acidophilus SD65 + L. casei SD07 + L. plantarum SD02. CK: control healthy conditions; IM: immune-attacked (chemo); L: chemo + 3L-low dose; M: chemo + 3L-middle dose; H: chemo + 3L-high dose. Predominant genera present in the group are shown in bold. * shows genera increased in $\mathrm{CK}$ and/or H.

${ }^{\circ}$ shows fungal genera specifically increased by $3 \mathrm{~L}$ M-dose in chemotherapy conditions. 
The heatmap analysis also highlighted two genera in particular, Fusarium and Pichia, highly abundant in feces from control and high lactobacillus-treated groups or repeatedly found in control samples but significantly altered by chemotherapy (Figure $3 \mathrm{AB} \&$ Table 1). Furthermore, correlation was observed between Rhizopus and Lactobacillus treatment. Rhizopus-levels were significantly altered by chemotherapy, but increased when Lactobacillus was added to phosphamide. High abundance of Rhizopus microbes was detected in H, M and L samples. Higher abundance of Rhizopus was found in M samples (CTX + middle dose $/ 2.5 \mathrm{ml} / \mathrm{kg}$ bodyweight of Lactobacillus, Figure $3 \&$ Table 1), suggesting a dose-effect relationship between the dose of bioproduct and the increase of specific microbes. We noted that high-, medium- and low-dose injections of $3 \mathrm{~L}$ cocktail were beneficial in reducing the levels of Acaulium, Mucor, Olpidium, Penicillium, Periconia, Xerochrysium and Xeromyces (Figure 3 \& Table 1). In heatmap, only one microbial genus (Phallus) was induced by chemotherapy and not cured by high dose of 3L (Figure 3A). However, medium and low doses of 3L were both able to eradicate Phallus fungi despite CTX treatment (Figure 3A \& Table 1). Phallus infection was not predominant in all IM samples (Figure 3B). This also showed the importance of dosage and/or a process of gradually decreased dose adjustment over time for a useful therapeutic application of $3 \mathrm{~L}$ treatment.

Effects of CTX and CTX + Lactobacillus therapy on gut microbiome. The microbial composition distribution histograms of each sample (CK-L) were shown at phylum, order, class, family, genus level separately (Figure $4 \&$ Table 2). The dominant microbial phyla were similar between control conditions and specific treatments (Figure 4A \& Table 2). However, CTX induced a significant decrease of Ascomycotalevels, which was not observed using high-doses of 3L during chemotherapy (Figure 4A). This beneficial effect of $3 \mathrm{~L}$ injections was observed for many other microbial fungal phyla, including Basidiomycota, Kickxellomycota and Mortierellomycota (Figure 4A \& Table 2). Levels of Mucoromycota and Olpidiomycota in rat fecal microbiomes were increased during CTX chemotherapy, but kept low when adding H, M or L doses of Lactobacillus to anti-tumor treatment (Figure 4A \& Table 2). Similarly, specific beneficial effects of Lactobacillus treatment in complement of chemotherapy for cancer were seen by analysis of the distribution of microbial fungal classes, orders, families and genera in the five rat groups (Figure 4B-E \& Table 2). The most abundant microbial fungal classes of healthy control rats without any 
other treatment than normal saline injection were Dothideomycetes, Eurotiomycetes, Saccharomycetes, Sordariomycetes and Tremellomycetes. All the five classes were seriously affected by chemotherapy. Chemotherapy also induced higher levels of Mucoromycetes in the fecal microbiome. Dothideomycetes, Eurotiomycetes and Sordariomycetes were conserved at normal levels with high dose injections of 3L. Importantly, Mucoromycetes were maintained at normal levels in all two Lactobacilli-treated samples. Other microbial classes such as Agaricostilbocytes, Leotiomycetes and Tremellomycetes, were also retrieved at normal levels upon probiotic treatments (Figure 4B \& Table 2). The most abundant microbes on order level were Eurotiales, Hypocreales, Saccharomycetales and Trichosporonales not only in CK, but also in $\mathrm{H}$ group. The microbial order profiling was different in IM group, with significantly altered levels of Eurotiales, Hypocreales, Saccharomycetales and Trichosporonales, as well as significantly increased levels of Mucorales. Capnodiales, Cystofilobasidiales and Glomerellales that were affected by chemotherapy were held to normal levels by Lactobacillus treatment. H, M and L doses of Lactobacillus were efficient in controlling the levels of Mucorales. Low dose of Lactobacillus was also particularly efficient in stimulating the levels of Mallasseziales, emphasing about the need of controlling the dose of Lactobacillus to target specific microbial orders (Figure 4C \& Table 2). Aspergillaceae, Didymellaceae, Nectriaceae and Pleosporaceae were four major microbial families identified in the rat fecal samples related to cancer and specific therapy. Interestingly, these microbial families were vulnerable to chemotherapy alone, but maintained by adding $3 \mathrm{~L}$ to CTX treatment. A gradual increase of 3L was necessary to maintain the levels of Aspergillaceae. Cordycipitaceae, Mycosphaerellaceae, Rhizopodaceae, Thermoascaceae and Trichosporonaceae-levels were also reduced by CTX but maintained when CTX was combined with Lactobacillus injections. It was not the case for all microbial families identified in rat fecal samples. Pichiaceae were an example of microbial families down-regulated after CTX treatment and immune-damage, which could not be reversed by adding Lactobacillus during treatment. However, Lactobacillus at high, medium and low dose had clear beneficial effects for the control of Mucoraceae. Mucoraceae-levels were very high in IM group, which could be reversed by adding $\mathrm{H}, \mathrm{M}$ or L dose of Lactobacillus. Low dose of bioproduct was found to induce particularly high levels of Mallasseziaceae (Figure 4D \& Table 2). Accordingly, Alternaria, Aspergillus, Fusarium and Mycosphaerella were the main microbial genera characteristic of CK and H groups, while Mucor was a diagnosis of immune-damage by CTX treatment. Very importantly, Mucor was controlled by adding 
Lactobacillus to chemotherapy. Very low Mucor-levels were detected in H, M and L groups of rats related to cancer and chemo/Lacto treatment. Similar effects were observed on Xerochrysium. Xerochrysium-levels increased during chemotherapy, but were controlled by using Lactobacillus as low/medium/high dose. Other microbial genera such as Chlamydomyces, Lecanicillium, Rhizomucor and Sarocladium altered by chemotherapy were maintained by Lactobacillus either at low, medium or high doses. Whatever the dose of 3L bioproduct, only Pichia was not maintained by Lactobacillus treatment (Figure 4E \& Table 2).

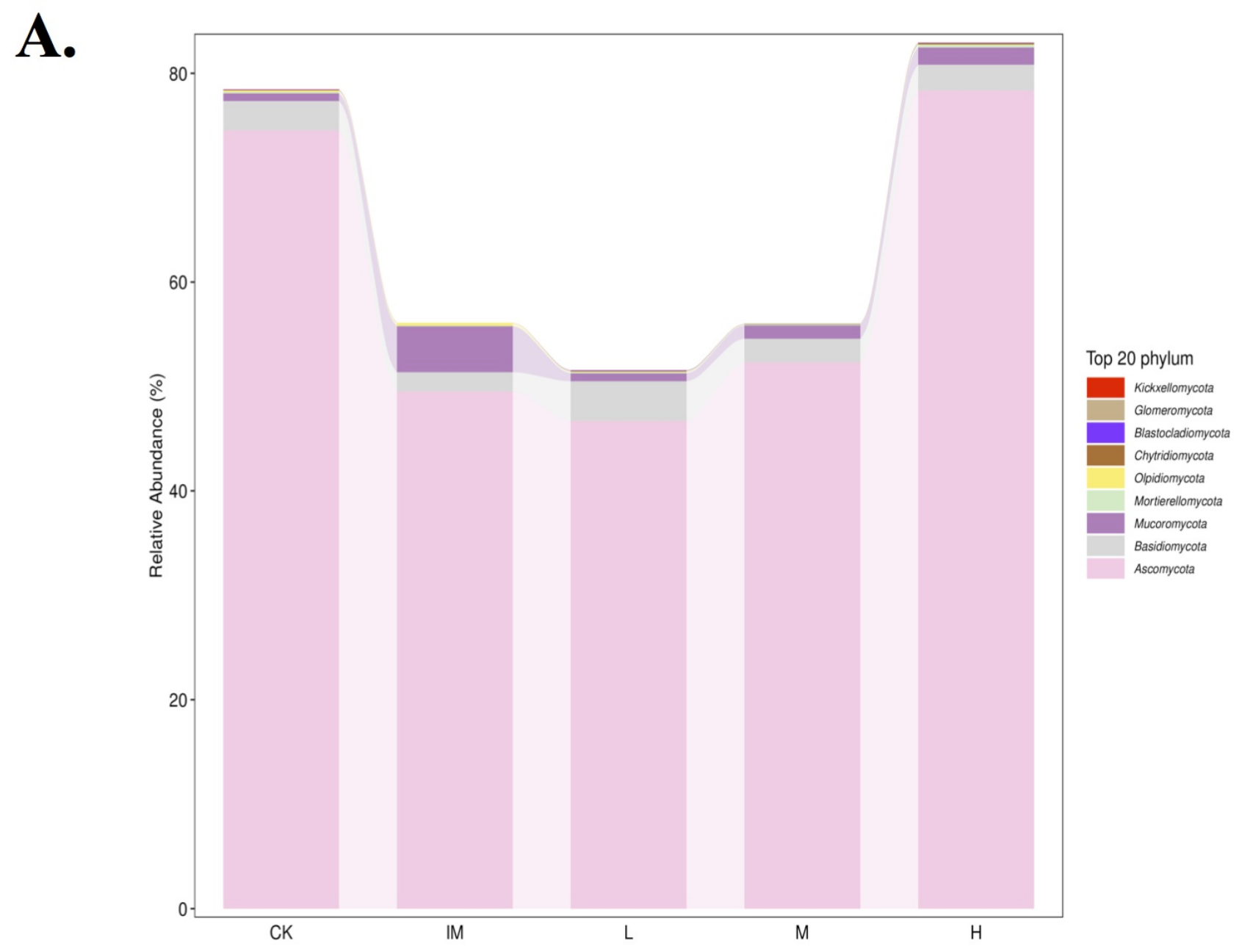


B.
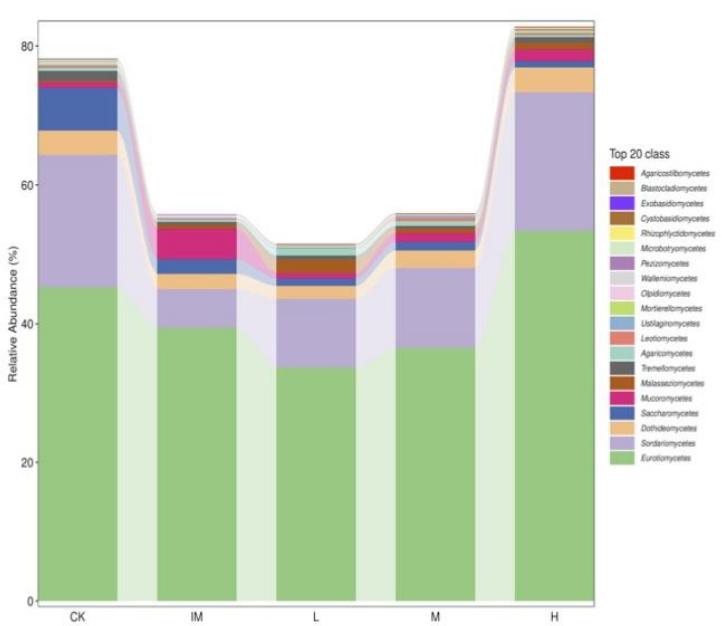

D.

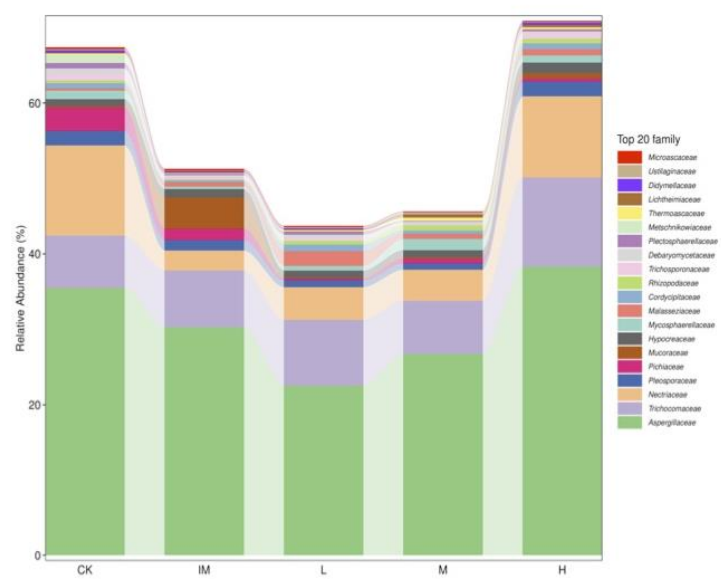

C.

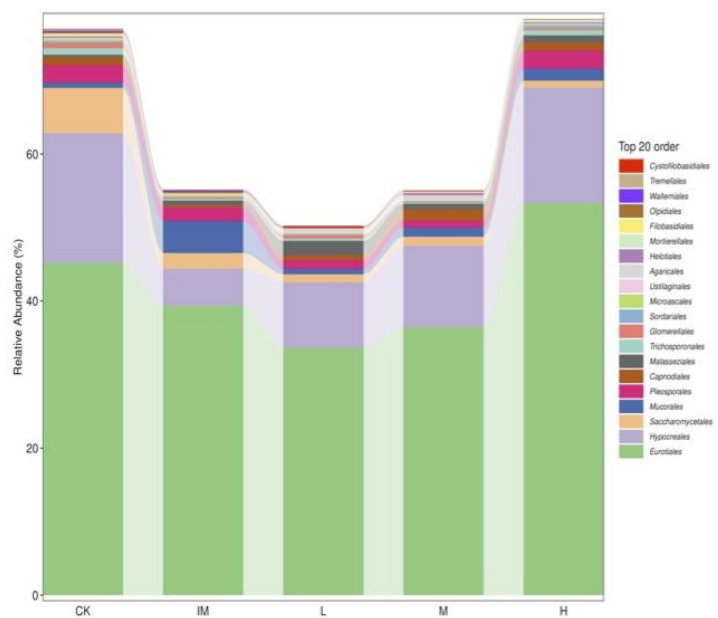

E.

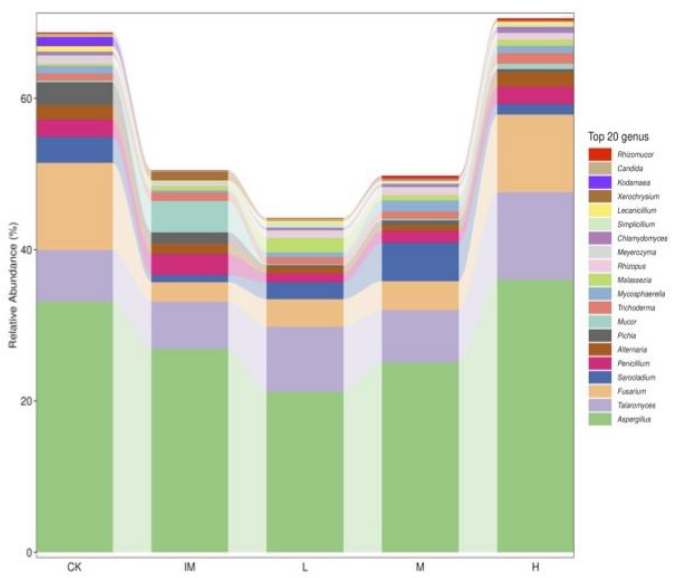

Figure 4 Bacterial taxa distribution in rat groups in relation with cancer and chemotherapy at phylum, order, class, family and genus level. A) Phylum-level. B) Class-level, C) Order-level, D) Family-level and E) Genus-level. The ratio or relative abundance of each bacterial taxa in a specific group is directly displayed (color code). At phylum-to-genus level, top 20 most abundant bacterial taxa in rat fecal samples were used to draw the histogram. The curve shows down-regulation of the rat gut flora after chemotherapy, but upregulation of the microbiome during high-dose Lactobacillus therapy (A-E, R(v3.1.1), BGI Co., Ltd). 


\begin{tabular}{|c|c|c|c|c|}
\hline CK & IM & $\mathrm{H}$ & $\mathrm{M}$ & $\mathrm{L}$ \\
\hline \multicolumn{5}{|l|}{ Phylum } \\
\hline Ascomycota & Ascomycota ${ }^{\circ}$ & Ascomycota & Ascomycota & Ascomycota \\
\hline Basidiomycota* & Basidiomycota $^{\circ}$ & Basidiomycota* & Basidiomycota* & Basidiomycota* \\
\hline \multicolumn{5}{|l|}{ Blastocladiomycota } \\
\hline \multicolumn{5}{|l|}{ Chytridiomycota } \\
\hline \multicolumn{5}{|l|}{ Glomeromycota } \\
\hline Kickxellomycota & $\mathrm{Kickxellomycota}^{\circ}$ & Kickxellomycota & Kickxellomycota & Kickxellomycota \\
\hline Mortierellomycota* & Mortierellomycota $^{\circ}$ & Mortierellomycota* & Mortierellomycota* & Mortierellomycota* \\
\hline Mucoromycota & Mucoromycota $\dagger$ & Mucoromycota & Mucoromycota & Mucoromycota \\
\hline Olpidiomycota & Olpidiomycota $\uparrow$ & Olpidiomycota & Olpidiomycota & Olpidiomycota \\
\hline \multicolumn{5}{|l|}{ Class } \\
\hline \multicolumn{5}{|l|}{ Agaricomycetes } \\
\hline Agaricostilbomycetes* & Agaricostilbomycetes $^{\circ}$ & Agaricostilbomycetes* & Agaricostilbomycetes* & Agaricostilbomycetes* \\
\hline \multicolumn{5}{|c|}{ 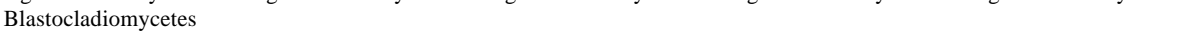 } \\
\hline \multicolumn{5}{|l|}{ Cystobasidiomycetes } \\
\hline Dothideomycetes & Dothideomycetes ${ }^{\circ}$ & Dothideomycetes & Dothideomycetes & Dothideomycetes \\
\hline Eurotiomycetes & Eurotiomycetes $^{\circ}$ & Eurotiomycetes & Eurotiomycetes & Eurotiomycetes \\
\hline \multicolumn{5}{|l|}{ Exobasidiomycetes } \\
\hline Leotiomycetes* & Leotiomycetes $^{\circ}$ & Leotiomycetes* & Leotiomycetes* & Leotiomycetes* \\
\hline \multicolumn{5}{|l|}{ Malasseziomycetes } \\
\hline \multicolumn{5}{|l|}{ Microbotryomycetes } \\
\hline \multicolumn{5}{|l|}{ Mortierellomycetes } \\
\hline Mucoromycetes & Mucoromycetes $\uparrow$ & Mucoromycetes & Mucoromycetes & Mucoromycetes \\
\hline \multicolumn{5}{|l|}{ Olpidiomycetes } \\
\hline \multicolumn{5}{|l|}{ Pezizomycetes } \\
\hline \multicolumn{5}{|l|}{ Rhizophlyctidomycetes } \\
\hline Saccharomycetes & Saccharomycetes $^{\circ}$ & Saccharomycetes & Saccharomycetes & Saccharomycetes \\
\hline Sordariomycetes & Sordariomycetes $^{\circ}$ & Sordariomycetes & Sordariomycetes & Sordariomycetes \\
\hline Tremellomycetes* & Tremellomycetes $^{\circ}$ & Tremellomycetes* & Tremellomycetes* & Tremellomycetes* \\
\hline \multicolumn{5}{|l|}{ Ustilaginomycetes } \\
\hline \multicolumn{5}{|l|}{ Wallemiomycetes } \\
\hline \multicolumn{5}{|l|}{ Order } \\
\hline \multicolumn{5}{|l|}{ Agaricales } \\
\hline Capnodiales* & Capnodiales $^{\circ}$ & Capnodiales* & Capnodiales* & Capnodiales* \\
\hline Cystofilobasidiales* & Cystofilobasidiales $^{\circ}$ & Cystofilobasidiales* & Cystofilobasidiales* & Cystofilobasidiales* \\
\hline Eurotiales & Eurotiales $^{\circ}$ & Eurotiales & Eurotiales & Eurotiales \\
\hline \multicolumn{5}{|l|}{ Filobasidiales } \\
\hline Glomerellales* & Glomerellales $^{\circ}$ & Glomerellales* & Glomerellales* & Glomerellales* \\
\hline Helotiales & & & & \\
\hline Hypocreales & Hypocreales $^{\circ}$ & Hypocreales & Hypocreales & Hypocreales \\
\hline Malasseziales & Malasseziales & Malasseziales* & Malasseziales* & Malasseziales* \\
\hline Microascales & & & & \\
\hline Mortierellales & & & & \\
\hline Mucorales & Mucorales $\uparrow$ & Mucorales & Mucorales & Mucorales \\
\hline Olpidiales & & & & \\
\hline Pleosporales & & & & \\
\hline Saccharomycetales & Saccharomycetales $^{\circ}$ & Saccharomycetales & Saccharomycetales & Saccharomycetales \\
\hline Sordariales & & & & \\
\hline Tremellales & & & & \\
\hline Trichosporonales & Trichosporonales $^{\circ}$ & Trichosporonales & Trichosporonales & Trichosporonales \\
\hline Ustilaginales & & & & \\
\hline Wallemiales & & & & \\
\hline
\end{tabular}

Table 2. Composition of the mycobiome (Phylum, Class, Order, Family and Genus of fungi and yeasts) identified in five rat groups in relation with cancer, chemotherapy and Lactobacillus treatment. Lactobacillus treatment: tritherapy $(3 \mathrm{~L})=$ L. acidophilus $S D 65+$ L. casei $S D 07+$ L. plantarum SD02. CK: control healthy conditions; IM: immune-attacked (chemo); L: chemo + 3L-low dose; M: chemo + 3L-middle dose; H: chemo $+3 \mathrm{~L}$-high dose. Predominant genera present in the group are shown in bold. * shows microbes significantly increased in CK and the three group doses of Lactobacillus (L, M and H).

$\circ$ shows microbes significantly decreased by chemotherapy. $\dagger$ shows microbes increased in chemo (see IM), but not in chemotherapy + Lactobacillus conditions (see L, M and $\mathrm{H}$ ). 
Comparing all the triplicates (Figure S9), Ascomycota-levels were found to be remarkably high in CK and $\mathrm{H}$ triplicates (Figure S9A). In contrast, particularly low levels of Ascomycota were found in IM021, L103 and M201. Mucoromycota and Olpidiomycota were extremely high in IM015 samples (Figure S9A). Both Mucoromycota and Olpidiomycota were much lower in Lactobacillus samples, in particular in H (H105, H204 and H205). Mortierellomycota were absent from IM triplicates (IM015, IM021 and IM024), but maintained in C101, C103, L104, M202, M203, H105, H204 and H205 (Figure S9A). In the analysis of ungrouped samples, high levels of Sordariomycetes, Saccharomycetes and Tremellomycetes were specific to control triplicates (C101, C103 and C105). High levels of Sordariomycetes and Tremellomycetes were maintained in L102, L104, M202, M203, H105, H204 and H205. IM021 was characterized by very low levels of Eurotiomycetes. IM015 was characterized by very high levels of Mucoromycetes. H samples all had Eurotiomycetes and Mucoromycetes maintained to normal conditions (Figure S9B). IM015 was characterized by high Mucorales/low Saccharomycetales on the order level (Figure S9C). However, although Saccharomycetales remained low in all different samples of H, M and L Lactobacillus-treatment, Lactobacillus samples all had levels of Mucorales similar to controls (Figure S9C). In addition, medium and high Lactobacillus samples M202-H205 had levels of Capnodiales similar to those measured in C101, C103 and C105. Both control (C101 and C103) and Lactobacillus (L104) had high Glomerellales-levels (Figure S9C). The profiling of microbial families was diverse in all different samples, but mainly in CK and $\mathrm{H}$ group. C101, C103, C105, H105, H204 and H205 all six diplayed high levels of Nectriaceae and Trichosporonaceae, among many other families from Aspergilaceae to Microascaceae. Importantly, none of the Lactobacillus samples showed the high levels of Mucoraceae observed in IM015 (Figure S9D). IM015 showed high levels of Mucor in the genus taxa summary from ungrouped samples, while C101, C102, C105, L102, L104, M202, M203, H105, H204 and H205 showed high levels of Fusarium, but no Mucor to the extent observed in the immune-damage sample IM105 (Figure S9E). Therefore, analyzing ungrouped samples of rat fecal microbiomes related to cancer and the effects of specific bioproduct therapy also argued for the very beneficial role of Lactobacillus in maintaining host health microbiome.

The metagenome sequence data analysis (CK versus $\mathrm{M}$ ) showed a pattern overlapping with enriched core microbes in the order Trichosporonales and in the phylum Basidiomycota (Figure S10). Relative abundance of Fungi, Ascomycota, Sordariomycetes, Hypocreales, Nectriaceae, Fusarium was very high 
(above 60000-140000) in CK and H class of samples. Relative abundance of Fusarium fungi was below 50000 in IM, L and M class of samples (Figure S11A). LEfSe (LDA, Krustal-Wallis and Wilcoxon) identified Fusarium as a key biomarker (i.e, a key community member) of CK group (Figure S11B). Comparative metagenomics and network analysis at phylum level showed a high degree of similarity between control and Lactobacillus-treated rat fecal samples and revealed that Ascomycota were dominant in this network (Figure S12). IM samples (in blue) are not associated to this CK-Lactobacillus group (Figure S12A). Mucoromycota (in orange) were dominant in CTX- immune-attacked ill rat fecal samples (Figure S12B).

Effects of CTX and CTX + Lactobacillus therapy on metabolic pathways. The relative abundance of each functional category (biosynthesis, degradation/utilization/assimilation/, generation of precursor metabolite and energy, glycan pathways and metabolic clusters) was estimated as pathway abundance and relative abundance of read counts (Figure S13). Differential abundance was mainly found for respiration, fermentation, fatty acid/lipid/carbohydrate degradation and biosynthetic pathways (Figure S13A). Similarly, raw counts for metabolic pathways and enzyme, metabolites and reaction orthologs in MetaCyc showed a strong statistical significance of differential abundance mainly for cofactor, prosthetic group, electron carrier, vitamin, fatty acid and lipid biosynthesis (Figure S13B). Some specific metabolic pathways in MetaCyc database can be labeled even with a light-level bacterial taxon [76]. Therefore, we also used MetaCyc to identify metabolic pathways and/or bacterial taxa specifically related to the five groups of rats for cancer therapy (Figure 5). MetaCyc L-methionine salvage cycle III was linked to a high Clostridiales-level in CK control group (PWY-7527, Figure 5A). Another pathway (PWY-7839), 6-hydroxymethyl-dihydropterin diphosphate biosynthesis I, which converts GTP into pterin precursors (methanopterin and sarcinapterin) for the biosynthesis of several cofactors in specific bacterial strains, was found to be particularly highly expressed in CK and Lactobacillus-treated samples due to not only to increased Lactobacillus-levels, but also to an increase in S24-7 Muribaculaceae, Prevotella, Clostridiales, Bacteroides and CF231 Paraprevotellaceae (Figure 5B). The anaerobic pathway for oleate biosynthesis IV (PWY-7664) was stimulated mainly by high doses of Lactobacillus. M- and H-doses of Lactobacillus significantly increased Prevotella- and Bacteroides-levels in rats subject to chemotherapy (Figure 5C). Similar beneficial effects of 
M- and H-doses of the bioproduct were found for mycolate biosynthesis (PWYG-321), and in particular, high levels of Bacteroides accumulated in M-treated samples (Figure 5D). Treatments with M- and H-doses were also particularly relevant to increase the levels of Bacteroides and Prevotella which were both essential in the pyridoxine pathway required for vitamin B6 synthesis (PYRIDOXSYN-PWY, pyridoxal 5'-phosphate (PLP) biosynthesis I; Figure 5E). In addition, abundance of helicobacterial taxa required for the TCA cycle (tricarboxylic acid cycle) or the Krebs cycle was also (re)-established by Lactobacillus treatment. Not only Helicobacter-levels, but also the levels of Flexispira, Rothia and Halomonas were maintained despite chemotherapy using Lactobacillus treatment (Figure 5F). Only Bacillales were not maintained by L-, M- or H-doses of Lactobacillus. Higher doses (> $5.0 \mathrm{ml} / \mathrm{kg}$ ) of $3 \mathrm{~L}$ bioproduct were probably required to control Bacillales. High dose $(5.0 \mathrm{ml} / \mathrm{kg})$ of $3 \mathrm{~L}$ was required to control Halomonas (Figure 5F). Importantly, a formaldehyde oxydation peak was noticed in IM samples. This was due to the development of Enterococcus pathogens in immune damage conditions (Figure 5G). Enterococcus infection was completely removed by adding Lactobacillus to chemotherapy (Figure 5G). Beneficial effects of Lactobacillus treatment during chemotherapy (+CTX) were also observed on TCA-GLYOX-BYPASS, the superpathway or bypass that integrates the common prokaryotic Krebs cycle (TCA) with the glyoxylate shunt. A high diversity of bacterial taxa was observed in CK and H-dose conditions. Enrichment of Enterobacteriaceae, Rothia, Cupriavidus, Halomonas and Devosia was detected in controls and persisted during CTX chemotherapy when using an additive probiotic treatment with high doses of 3L, altough treatment with Lactobacillus was inefficient on Bacillales at $1.25-5 \mathrm{ml} / \mathrm{kg}$ doses (Figure $5 \mathrm{H})$. Medium-dose $(2.5 \mathrm{ml} / \mathrm{kg}$ ) of Lactobacillus was particularly efficient in the control of Enterobacteriaceae (Figure 5H). Similarly, Bacteroidales, Bacteroides, Enterobacteriaceae, Halomonas and Devosia responsible for (prokaryotic) TCA cycle I was positively regulated by the additive Lactobacillus treatment (Figure 5I). A positive effect of $3 \mathrm{~L}$ bioproduct as an additive to chemotherapy was also observed by analysis of bacterial strains involved in the pathway teichoic acid (poly-glycerol) biosynthesis, which is part of cell wall biogenesis. Lactobacillus helped maintain Clostridiales, Mogibacteriaceae, Ruminococcaceae and Gemella to low levels, while strains such as Jeotgalicocaccus were stimulated (Figure 5J). No particular bacterial strains were identified for the superpathway of L-theronine metabolism, except for enterobacter in some low-dose Lactobacillus samples (Figure 5K). In contrast, tRNA charging was strongly affected by the CTX chemotherapy and/or the treatment 
with $3 \mathrm{~L}$ bioproduct. Using high doses of Lactobacillus, in particular, we had observed the main stimulatory effects of 3L on S24-7, Prevotella, Bacteroides, Ruminococcus, CF231 and Oscillospira (Figure 5L). Finally, Illumina and MetaCyc analysis found a strong effect of a middle dose of Lactobacillus on Enterobacteriaceae that mediate the bacterial superpathway of coenzyme Q ubiquinol-8 biosynthesis (UBISYN-PWY, Figure 5M). Lower effects were found for Cupriavidus and Stenotrophomonas (Figure 5M). In the pathway UDPN-acetyl-D-glucosamine biosynthesis I (UDPNAGSYN-PWY), CTX and immune-attacked (IM) strongly damaged the levels of Clostridiales, Ruminococcaceae, Ruminococcus and Oscillospira, which could be cured by using a high dose $(5 \mathrm{ml} / \mathrm{kg}$ ) of Lactobacillus bioproduct in complement of CTX (Figure $5 \mathrm{~N}$ ).

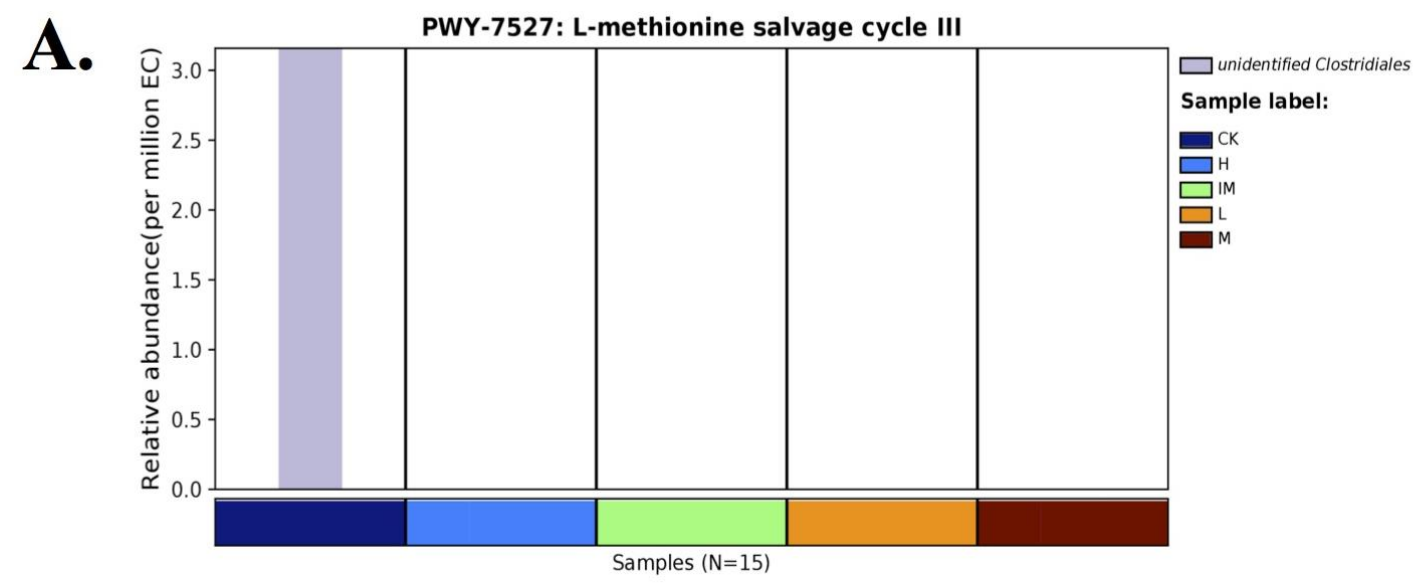

B.

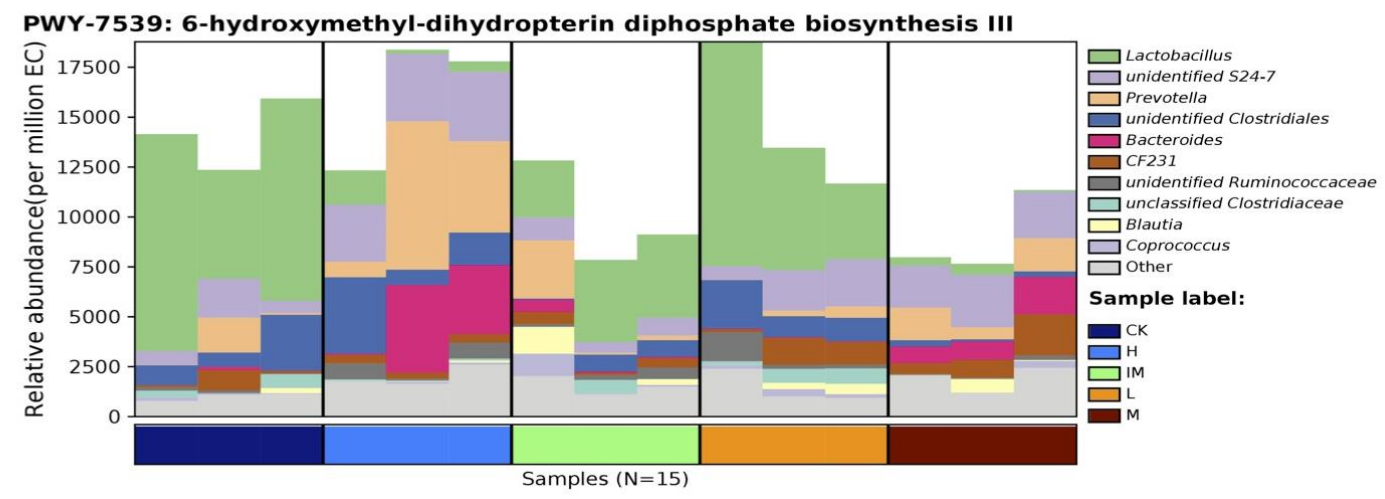


C.

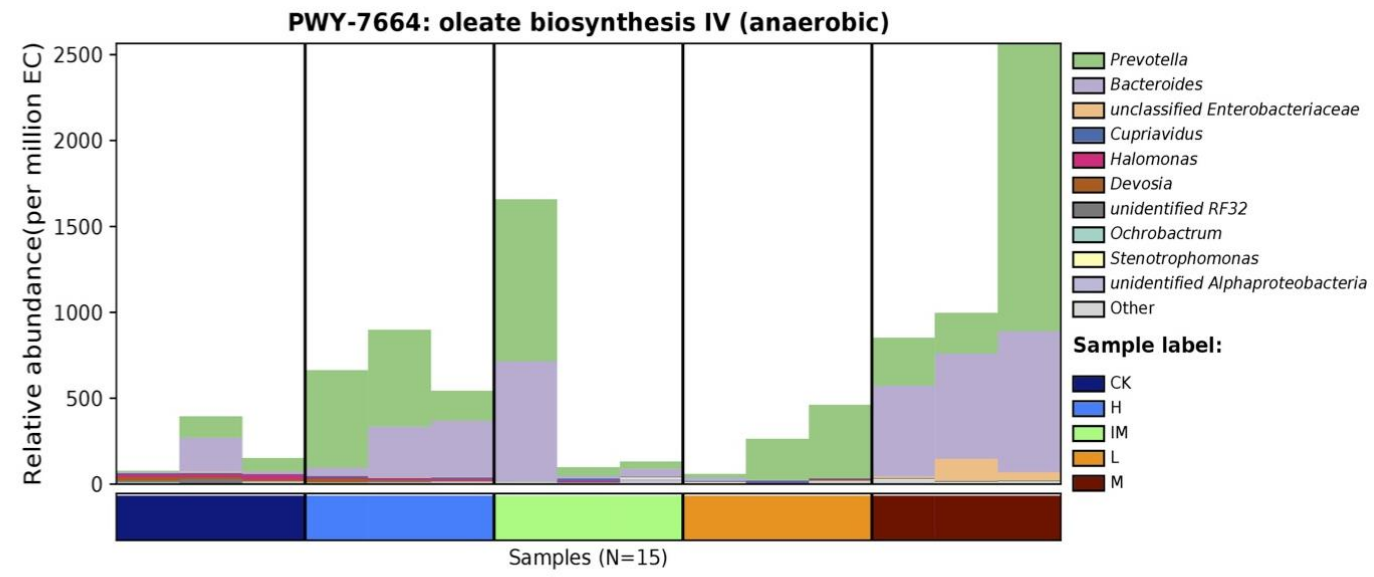

D.

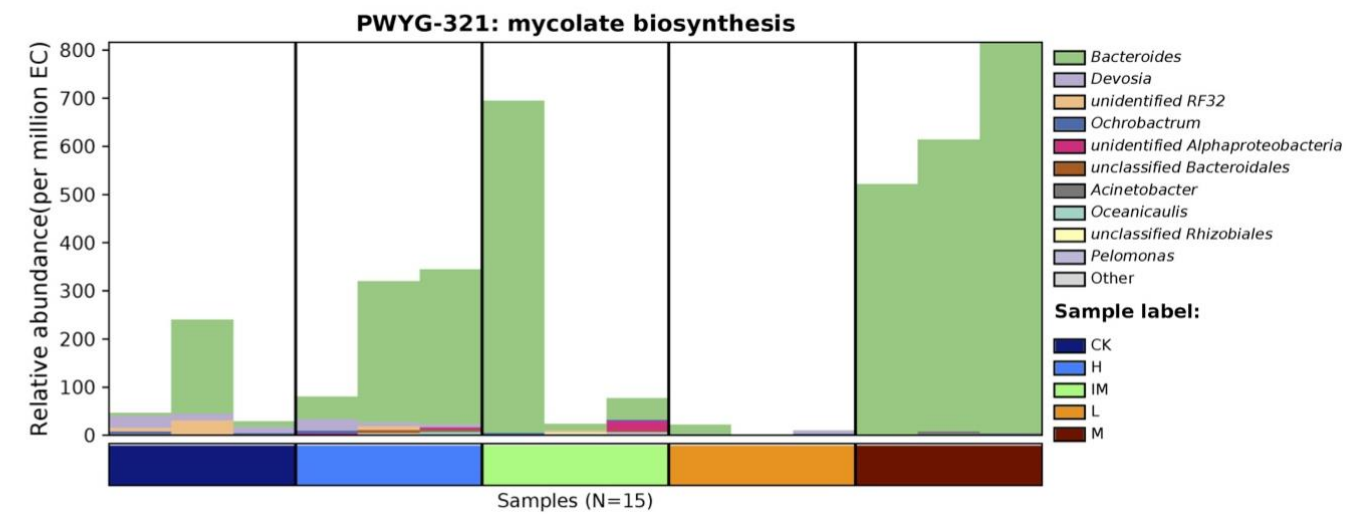

E.

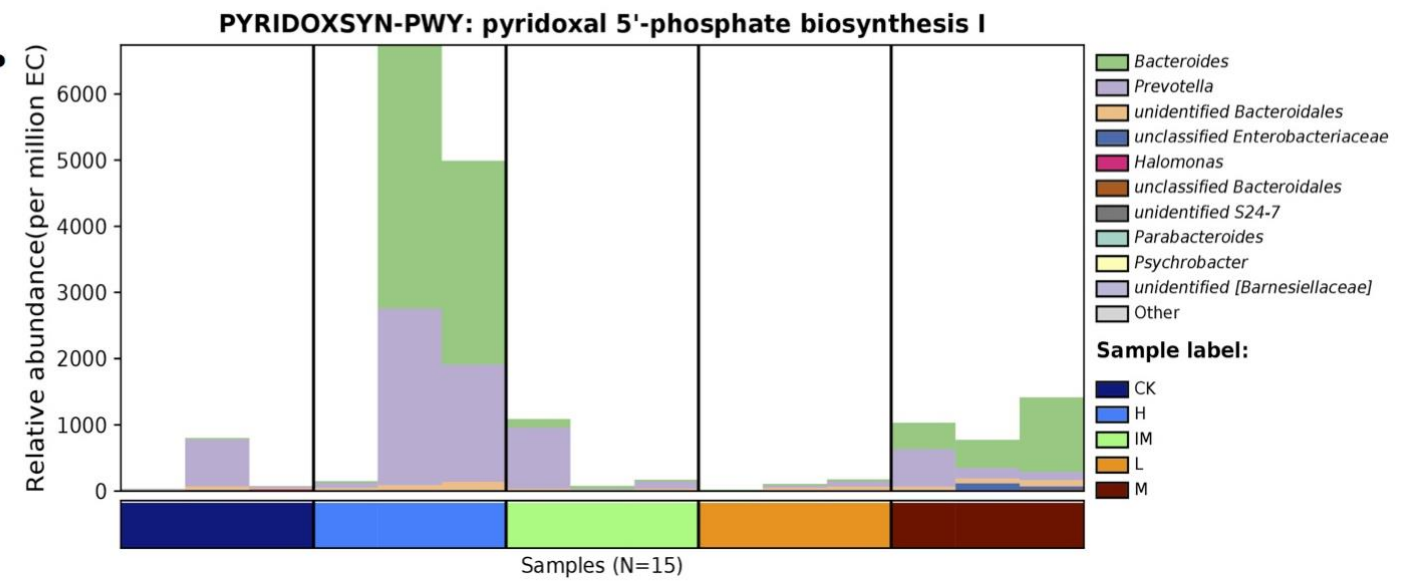

F.

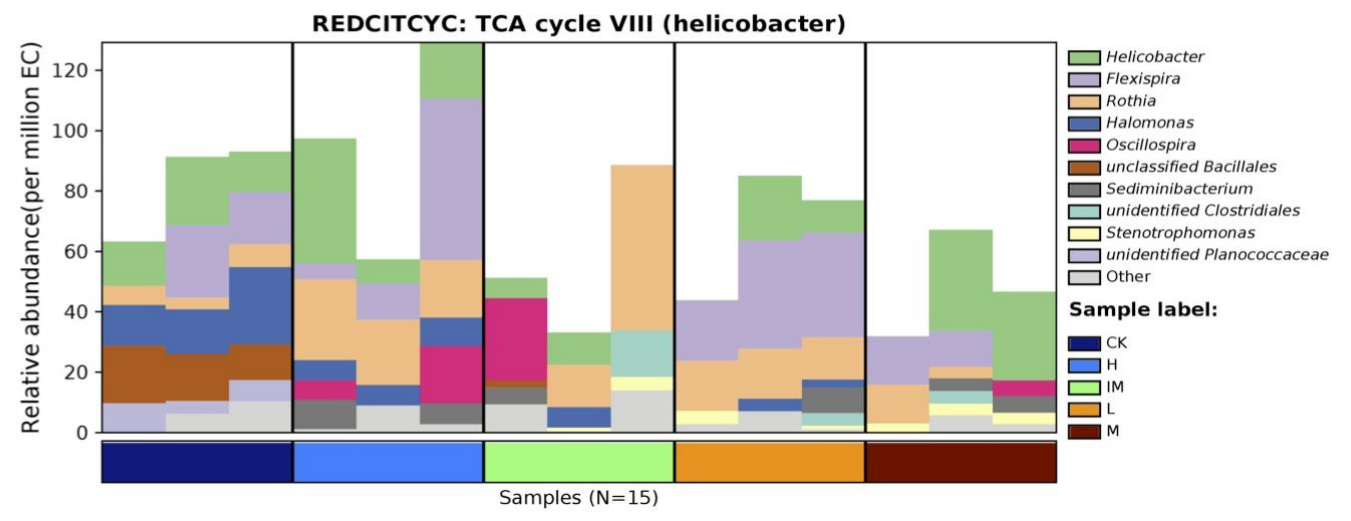




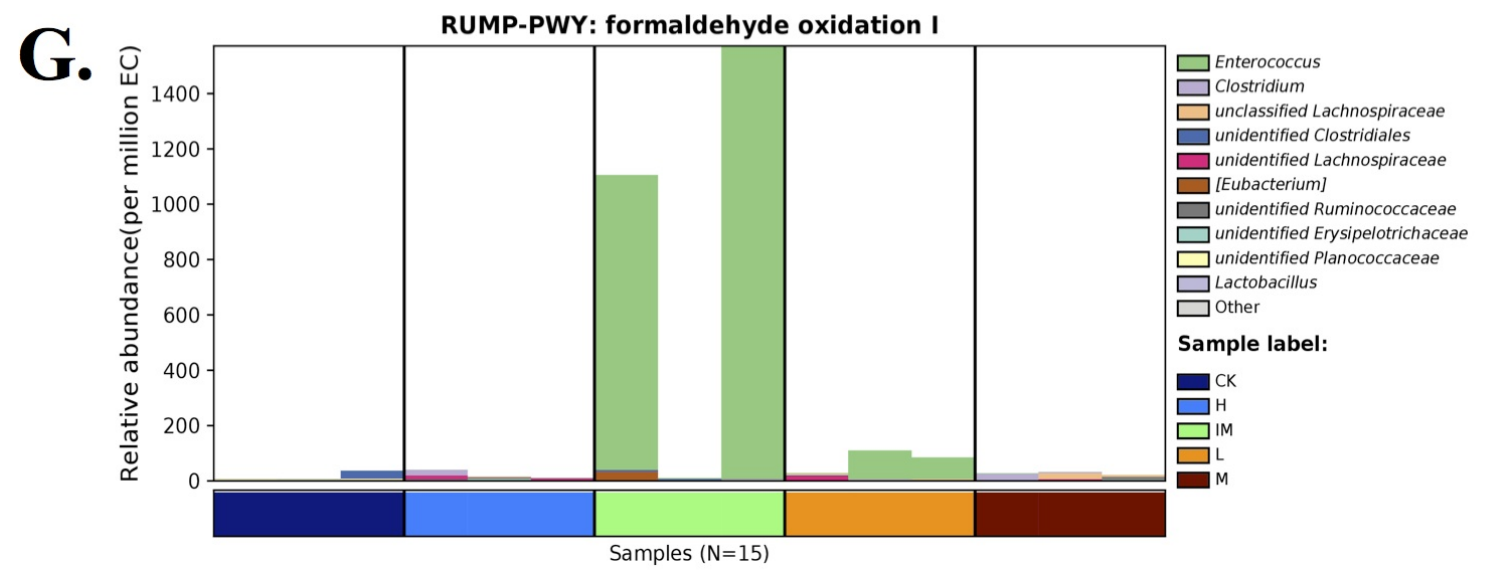

H.

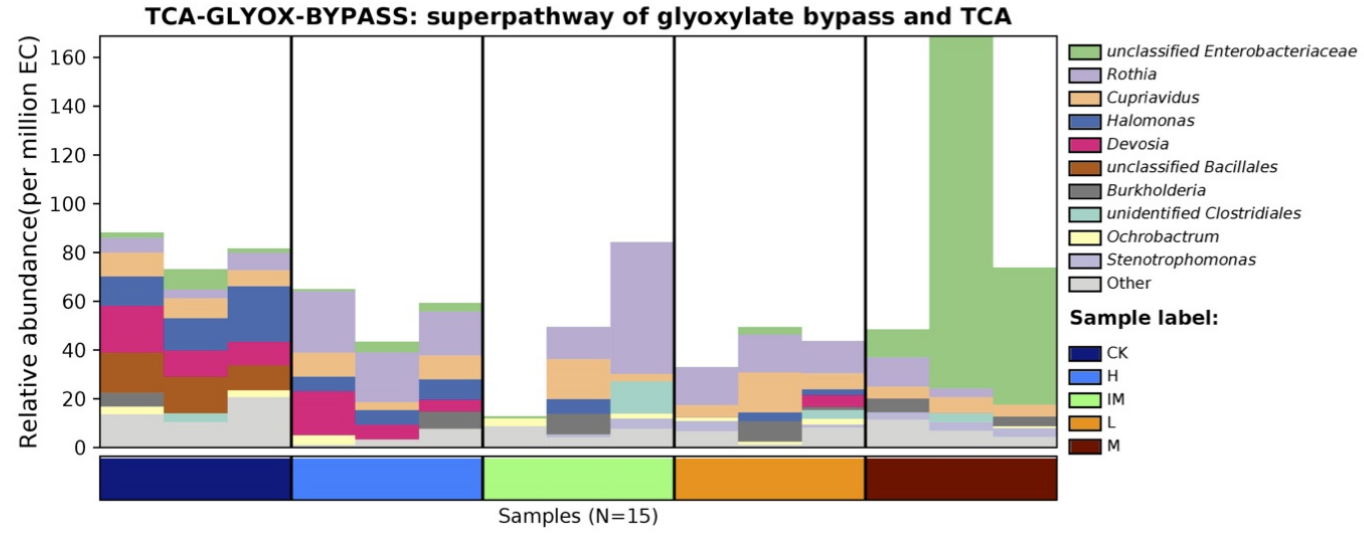



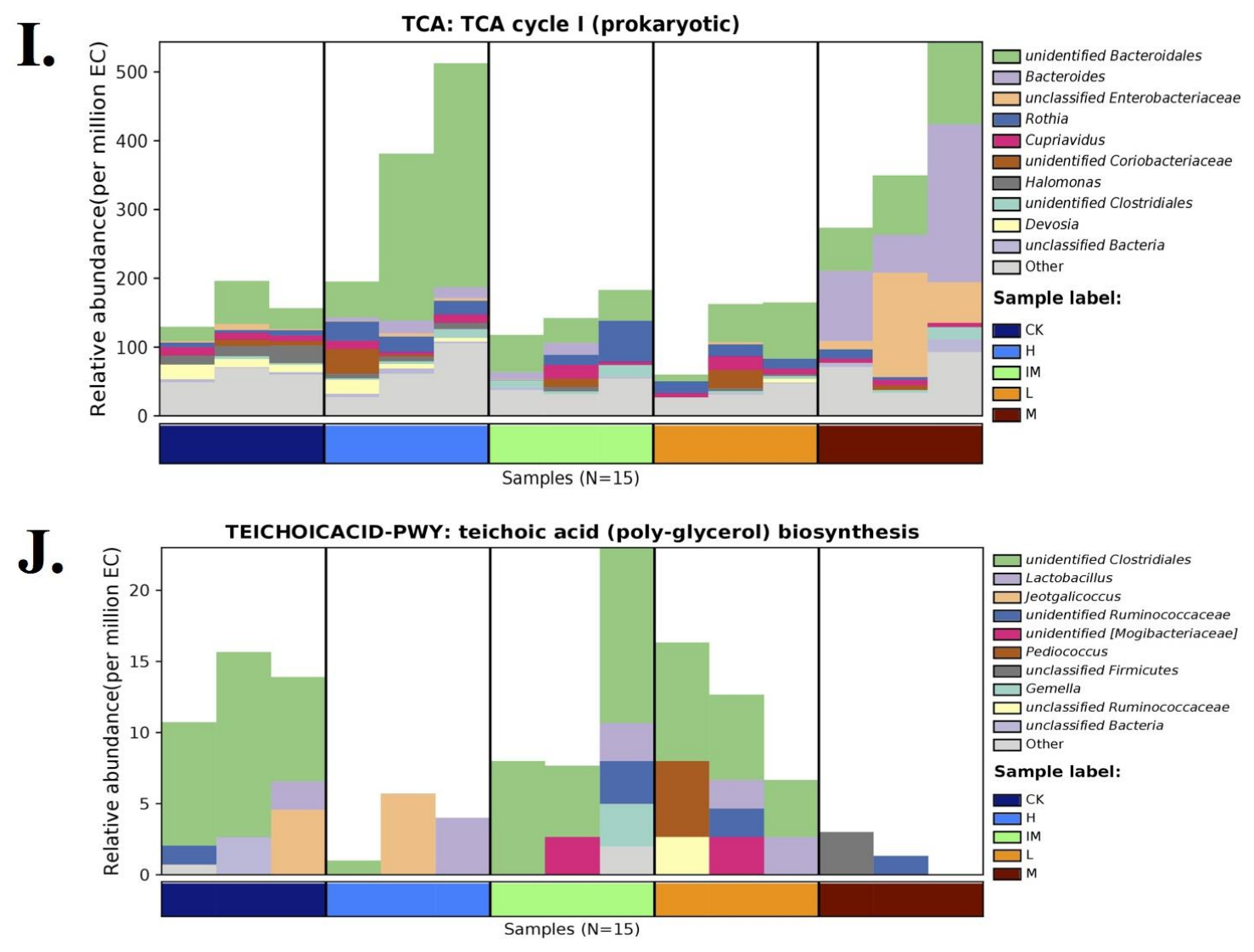
K.

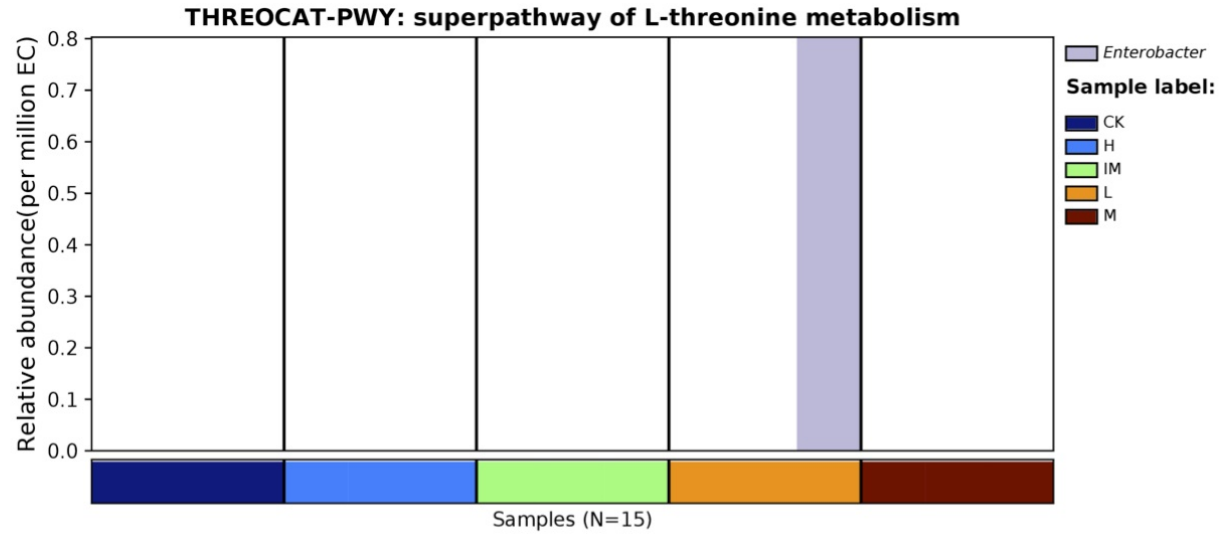

L.

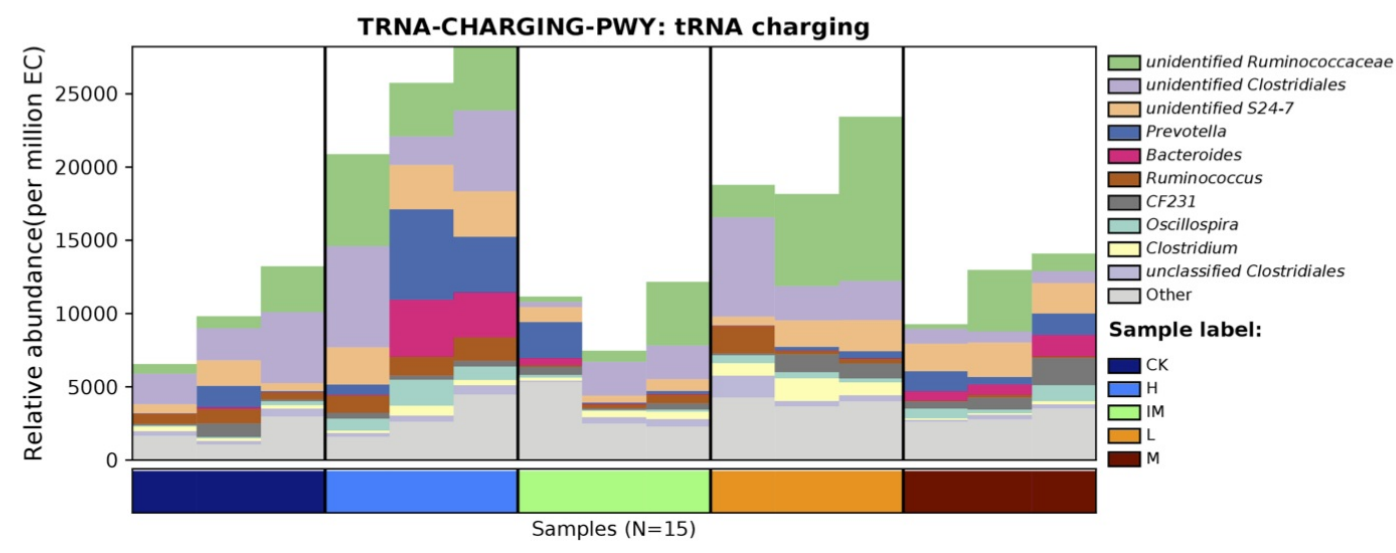




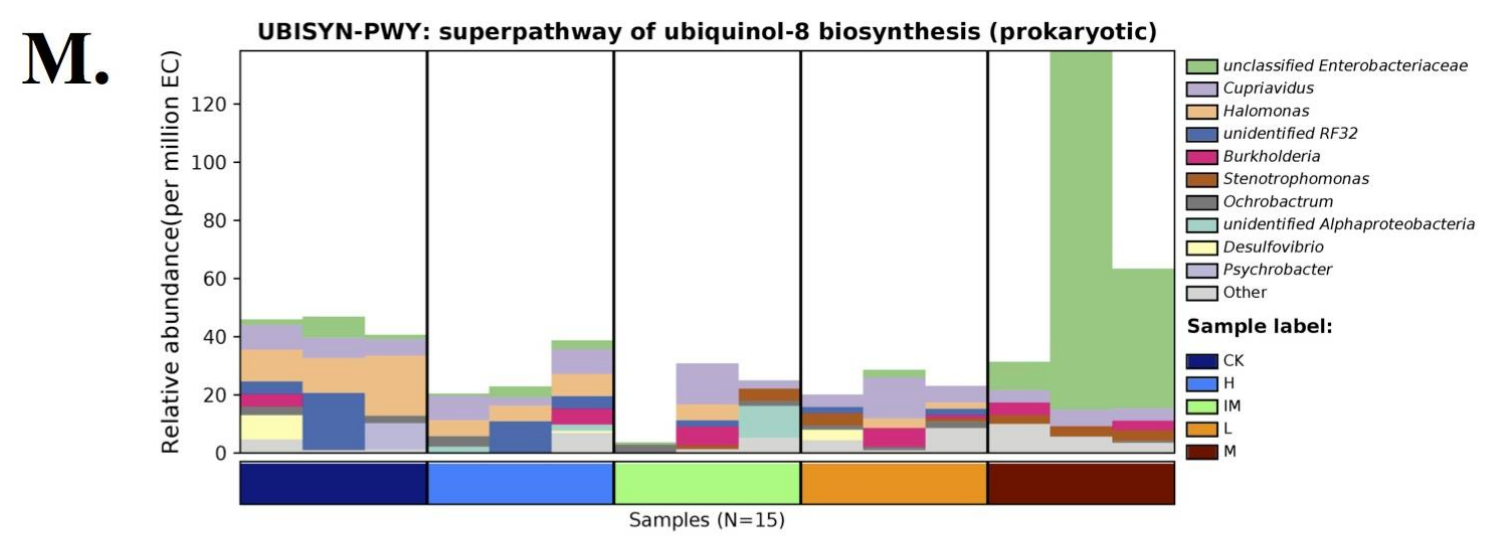

N.

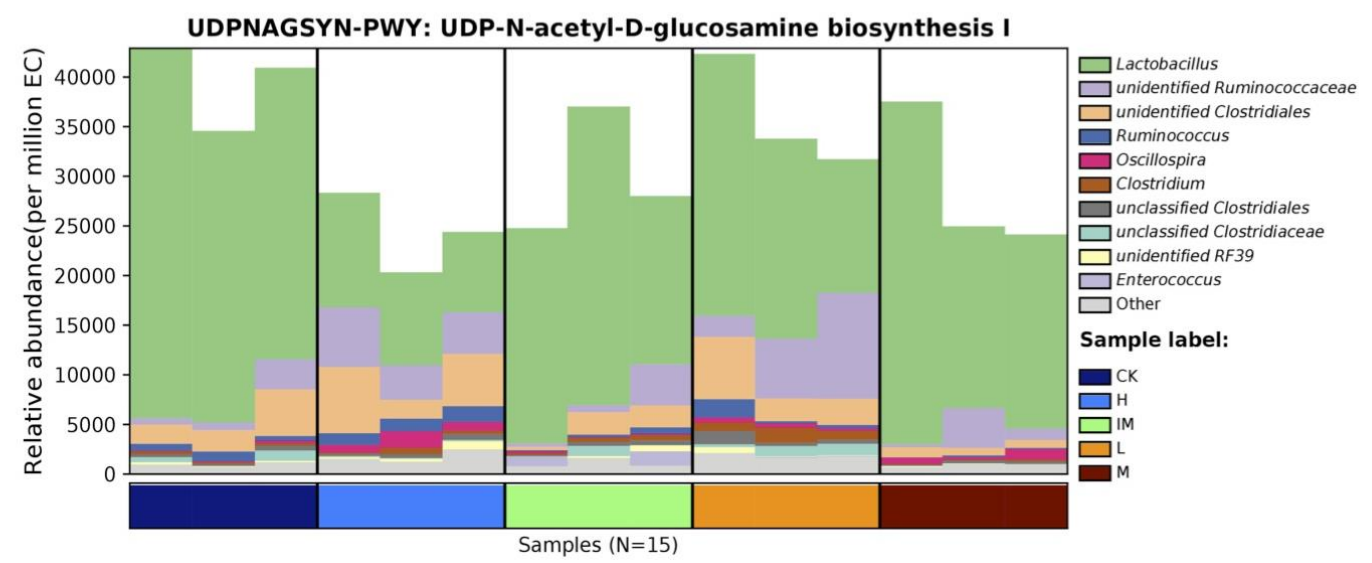

Figure 5 Regulation of metabolic pathways with bacterial taxa stratifications in rat groups in relation with cancer and chemotherapy. Three samples were tested per group. CK: normal (control healthy), IM: immunedamaged (ill), L: chemotherapy + 3L-low dose, M: chemotherapy + 3L-middle dose, H: chemotherapy + 3Lhigh dose. A) L-methionine salvage cycle III, B) 6-hydroxymethyl-dihydropterin diphosphate biosynthesis III, C) Oleate biosynthesis IV, D) Mycolate biosynthesis, E) Pyridoxal 5'-phosphatre biosynthesis I, F) TCA cycle VIII, G) Formaldehye oxidation I, H) Superpathway of glyoxylate bypass and TCA, I) TCA cycle I, J) Techoic acid (poly-glycerol) biosynthesis, K) Superpathway of L-threonine metabolism, L) tRNA charging, M) superpathway of ubiquinol-8 biosynthesis and N) UDP-N-acetyl-D-glucosamine biosynthesis I. The relative abundance of each bacterial taxa in a specific group is directly displayed (color code). The ordering method is based on the relative abundance of bacterial taxa and enzymatic acitivities (MetaCyc). High dose of Lactobacillus (H group) is ordered with control CK and healthy conditions for both metabolism and gut flora. 


\section{Discussion}

Cancer as cell disease caused by changes to DNA is a major burden of threat to human health worldwide. Even more burden of threat to human health comes when chemotherapy is envisioned as the primary or maybe sole way to prevent cancer development. Using one or more anti-cancer chemical drugs such as cyclophosphamide (cytophosphane, CTX) kills lymphoma or any cancer cells, but it kills or seriously alters also the patient immune system, challenging life and health expectancies perhaps just like the disease itself. This is clearly shown in our study when a strong beneficial healthy gut flora is suppressed by cancer and CTX treatment. Here, we have analyzed the microbiome of five groups of rats in relation with cancer, revealing the completely altered microbiome of rats treated by CTX. Very importantly, we show that lactobacillus treatments are particularly efficient to maintain the normal healthy control gut bacterial flora in the rat intestine and therefore able to establish strong healthy conditions in rats despite chemotherapy. Though there are earlier reports regarding the use of Lactobacillus in gut flora and gastrointestinal tract protection [77-80], we will always seek for the best solution and we still seek for a probiotic remedy with strong and significant impact on the adversive effects developed by CTX therapy, active on benefical microbes, improving microbial balance, activating nutrients and stimulating gut-powered immune systems.

Use of probiotics in cancer is still rare, not applicable to all ages and populations, an emerging field with many contradictory clinical results when comes to interaction with host or consumption by human patient [81-82]. Microbiota manipulation by natural probiotics vs chemical drugs is a constant challenge in human and veterinary medicine, in particular for genetic diseases such as cancer. The safety and stability of chemotherapeutic drugs such as CTX in clinical trials for cancer are doubtful [83-84]. We always look into clinical trials for cancer with high efficiency, with reduced resistance capacity and without affecting the quality of life and patient's health conditions. One has an ability to develop resistance to some chemicals. Repeated use of the same class of chemicals to control a disease such as cancer can cause many undesirable effects. When one becomes sick and resistant, the chemical (CTX) is used more frequently and the adjuvant must ultimately be added as CTX increases. We have not conducted yet a comprehensive microbiological medical study of the impact of $3 \mathrm{~L}$ on cancer. We have analyzed the rat response to a new bioproduct (3L: $L$. acidophilus + L. casei + L. plantarum) in addition to cyclophosphamide CTX. We have demonstrated that the association of acidophilus, casei and plantarum is particularly beneficial to maintain the gut flora and 
therefore the immune system during chemotherapy for cancer treatment. We present that connection of three Lactobacilli strains has strong beneficial effects on gut microbiota during chemotherapy.

Here, we present MiSeq data for the variations in the microbiome of rat feces under five different conditions: healthy normal, immune-attack (CTX chemotherapy), low, medium and high doses of Lactobacillus (3L), respectively, with special attention for fungal phylum, class, order, family, genus and species associated with cancer and its treatment with one anti-cancer cyclophosphamide drug. In this study, we demonstrate that rats treated with CTX + high doses of a new natural biomedical product (3L) have preserved microbiota of prime importance to sustain strong immune system and healthy condition. Such results are particularly important since it has been clearly established that bacterial dysbiosis accompanies carcinogeneis in malignancies as various as colon, liver and pancreas cancers. In particular, the growth of nocive fungi (Malassezia species) in the gut microbiome can promote oncogenesis via activation of mannose-binding lectins [85], which urges medication to control microbiome and MBL.

It is known that chemotherapy during cancer treatment adversely affects the composition of the gut flora, alters immune, metabolic and physiological functions, and potentially stimulates invasive fungal infection [86-88]. So it is not very surprising to find that CTX alters gut flora and provokes an increase in mucor or xerochrysium infection in groups of rats (see Figs. 3-4, S9-S12 \& Tables 1-2). The striking finding of our study in five groups of rats in relation with cancer and chemotherapy is the beneficial regulation of the gut flora after injection of different doses of Lactobacillus. High doses of 3L are shown to maintain normal gut flora in rats treated with CTX (see Figs. 2-5, S9-S12 \& Tables 1-2), urging to apply the method or lactobacillus treatment in human cancer therapy. We show here that high doses of L. acidophilus + L. casei + L. plantarum are particularly relevant to regulate the levels of Ascomycota and Capnodiales. This may be important discovery for cancer treatment due to the finding that ascomycetes are known to be used in medicine with the antibiotics penicillin and cephalosporin [89], while endophytic sooty mold fungi (Dothideomycetes) can be important for tissue health, environmental adaptation and stress tolerance [90]. Interestingly, high doses of $3 \mathrm{~L}$ ( $\mathrm{H}$ treatment) are shown to stimulate the levels of many various fungi families such as Aspergilaceae, Microascaceae, Nectriaceae and Trichosporonaceae, among others. Although many 
of these fungi are plant and human pathogens, many of them are known as biodegraders and biocontrol agents which could be exploited in medical applications [91]. For instance, this could be exploited for cancer treatment if we consider that these ascomycete fungi can help degrade residual CTX and control cyclophosphamide toxicity [92]. Another important aspect of using lactobacillus for cancer treatment is that 3L (high dose, $5.0 \mathrm{ml} / \mathrm{kg}$ ) is shown to keep pathogens such as mucormycetes (Mucormycota, Mucoraceae) and chytridiomycetes (Olpidiomycota, Olpidiaceae) at bay (see Figs. 4, S9-S12 \& Tables 1-2). High mucorales usually invade the blood vessels and are related to emerging infectious diseases such as mucormycosis beyond other zygomycoses [93]. Chytrid fungi cause chytridiomycosis, an emerging disease in amphibians, and a subcutaneous phycomycosis in humans [94]. Therefore, this is a complete pattern of many various fungal infections which can be regulated by 3L. Furthermore, 3L can control the magnitude of beneficial fungal components of the intestinal microbiota, i.e. the gut mycobiome in human health. Some fungi coming from the diet or the environment are important to mediate interaction in the gut bacterial communities and regulate metabolic homeostasis [see 72-73]. This is the case for Eurotiales, Hypocreales, Saccharomycetales and Trichosporonales that are crucial parts of healthy mycobiome in control and lactobacillus-treated rats (see Figs. 4, S9-S12 \& Tables 1-2). It could be important to use 3L to maintain the mycobiome balance, microbial community interactions, bacterial-fungal interactions, fungal-fungal interactions and host-fungal interactions during cancer treatment [95]. Similarly to diabetis [96], CTX injections resulted in significant changes in Mortierellomycota-levels, which could be reversed by adding L3 (see Fig. S9A). So, using L3 seems to be extremely useful to target specific components of the mycobiome that plays a key role in the development of many various diseases from cancer to diabetis.

In this prospect for curing or optimizing health, it is probably important to note that the effect of $3 \mathrm{~L}$ can be dose-dependent. Low dose of 3L cocktail $(1.25 \mathrm{ml} / \mathrm{kg})$ was required to treat the rats for Mallasseziomycetes (see Fig. 4C \& Table 2), which could be important in medications for specific pathologies such as colorectal cancer. Fungi included in the class Mallasseziomycetes increase with late-stage colorectal cancer [97]. During the phases of chemotherapy treatment in rats, we also find that $3 \mathrm{~L}$ doses work differently on the mycobiome. For threeL to be effective, quantities of lactobacillus ingredients eventually need to be gradually increased during cancer treament for a specific target fungal group, including Aspergillaceae, 
Cordycipitaceae, Mycosphaerellaceae, Rhizopodaceae, Thermoascaceae and Trichosporonaceae (see Fig. 4D \& Tables 1-2). Regulation of the fungal mycobiome is an important aspect of cancer treatment and chemotherapy. The gut mycobiome is implicated in microbiome assembly and immune functionality, which prompts the modulation of specific fungi to regulate both gut microbiome and immune system in therapeutic approaches for cancer [98]. However, while $3 \mathrm{~L}$ is effective to modulate yeasts in the order Saccharomycetales (Ascomycota; see Fig. 4 \& Table 2), Pichia is one of the rare examples of fungal genera that are affected by CTX chemotherapy but do not respond to lactobacillus treatments (see Fig. 4E \& Tables 1-2). A possible explanation might be that $3 \mathrm{~L}$ rather controls gut fungi, but not the oral fungi or genera such as Pichia and Candida [99]. Accordingly, a specific oral fungal medication needs to be prescribed to target Pichia and/or Candida. Perhaps the formulation of $3 \mathrm{~L}$ can still be improved to efficiently treat both oral and gut mycobiomes. Using one or more Lactobacillus strain such as L. reuteri in addition of 3L may be very effective to control the complete human mycobiome on oral and gut diseases (see Jørgensen et al., 2017 [100] \& our study).

However, one major striking finding of our study about Lactobacillus on cancer disease was the plethora of metabolic functions maintained by 3L treatment in five groups of rats in relation with CTX chemotherapy (see Figs. 5 \& S13). Not only cancer disease, but also chemotherapy heavily alters cell metabolism [101104]. Therefore, targeting of cancer (and chemotherapy) metabolism, as a complementary strategy, is a rather promising approach not only for therapeutic intervention on the disease, but also for preservation of physiological function in the patient immune system. We show here that a preparation of L. acidophilus (SD65), L. casei (SD07) and L. plantarum (SD02) is extremely efficient not in curing cancer, but in maintaining and/or stimulating many various metabolic pathways via a beneficial effect on the gut flora (see Figs. $5 \&$ S13). After treatment with our preparation of three lactobacilli cocktail (3L), the gut flora of rats subject to chemotherapy is rich in Firmicutes-Clostridia-Clostridiales-S24-7, as found for healthy control conditions (see Fig. 5). Overall, the detail composition of the bacteriome with the MetaCyc data show shared patterns of microbial strains and metabolic activities in healthy and 3L-treated groups for many various systems. Bacteroidales-Muribaculaceae-S24-7are important components of the microbiome for carbohydrate metabolism, while genes for amino acid and vitamin metabolism are upregulated in Deferribacteraceae [105]. 
Bacteroidales-Prevotellaceae-Prevotella-Paraprevotella plays a key role in the gastrointestinal tract for glucose (central carbon) metabolism, polysaccharide breakdown, glycogen storage, sulfate assimilation and production of propionate, which has anti-cancer and anti-inflammatory activities [106-109]. Campylobacterales-Helicobacteraceae-Helicobacter (pylori) is not necessarily infectious or associated with metabolic syndromes such as atherosclerosis. In many cases in humans, it can be rather considered as very beneficial to prevent the development of autoimmune diseases. H. pylori can help regulate fatty acid metabolism. It is also well known that $H$. pylori can contribute to the metabolic fate of pyruvate to lactateacetate, nucleotide biosynthesis, oxidative metabolism and therefore cellular respiration [110-112]. This dual aspect ("beneficial pathogen") shows the importance of our finding with $3 \mathrm{~L}$ maintaining control over helicobacter levels during cancer treatment (see Fig. 5D). This includes the potential of $3 \mathrm{~L}$ to control helicobacters of the genus Flexispira, urease-producing microorganism from the mid-colon and jejunum usually associated with diarrhea symptoms and complete febrile illness such as malaise, arthralgias, pain, leg swelling and polyserositis [113-114]. It also includes a beneficial control of 3L for ActinomycetalesMicrococcaceae-Rothia and Oceanospirillales-Halomonadaceae-Halomonas (see Fig. 5F), which might be important because Rothia and Halomonas are both prevalent in oral (salivae), oropharynx, respiratory tract and intestinal (gut) microbiota where they contribute to maintain healthy mucosal surfaces (iron scavenged from food, breakdown of proline and glutamine-rich proteins, glutamate and central carbohydrate metabolism) [115-116]. The stimulatory effects of 3L-pharmacological agents on central carbon metabolism and energy production are further illustrated by the beneficial effects of high doses of 3L (in complement to CTX for cancer) on the levels of Burkholderiales-Burkholderiaceae-Cupriavidus and RhizobialesHyphomicrobiaceae-Devosia (see Fig. 5H). Cupriavidus bacteria have a wide and diverse metabolic range that can be turned to sulfur-biofuels and energy sources from hydrogen and $\mathrm{CO}_{2}$ [117]. Similarly, rhizobium and Devosia are known to express multiple metabolic activities, including carbohydrate, cysteine, methionine, branch-chain amino acid and phosphorus compound metabolism, particularly suitable to sustain the synthesis of cellular and genetic components, energy transfer and/or mycotoxin degradation [118-119]. In addition, 3L mix can up-regulate the levels of Bacillales-Staphylococcaceae-Jeotgalicocaccus (see Fig. 5I-J), which is important for biotin/vitamin $\mathrm{H}$ or vitamin B7 metabolism, cofactor in carboxylase activities in the gut and its relation with human health [120]. This dozen bacterial families are upregulated by L3 treatment, emphasizing 
on the use of lactobacillus to stimulate amino acid, biotin, carbohydrate, glucose, iron, nitrogen, oxygen, phosphorus, protein, pyruvate, sulfide and vitamin metabolism as alternative or complement to chemotherapy for cancer (see Fig. 5). However, the most striking effect of Lactobacillus treatment on the microbiome is found for Enterococcus (see Fig. 5G-M). Enterococcus infection (bacteraemia, peritonitis, endocarditis and urinary tract infection) is significantly increased by chemotherapy (see Fig. 5G), which becomes a risk of ulcerative colitis or even a risk of colorectal cancer [121]. To raise bacteroides and clostridiales and keep Enterococcaceae at bay in order to balance the gut flora to normal healthy conditions during chemotherapy, we find that probiotics such as lactobacilli are very suitable (see Fig. 5). Different doses of lactobacillus are recommended for increase in levels of specific beneficial bacterial strains. M-doses are suitable for increase in levels of bacteroides, but higher doses of 3L are recommended to increase Halomonas-levels (see Fig. 5DF). Middle doses $(2.5 \mathrm{ml} / \mathrm{kg})$ of $3 \mathrm{~L}$ are also particularly suitable for decrease in levels of bacterial families commonly affecting the stomach and intestines such as enterobacteria (see Fig. 5H-M). So, not only intestinal and oral fungi (mycobiome) but also the complete bacterial microbiome can be controlled by $3 \mathrm{~L}$ in a strainand dose-dependent manner.

It is known that different formulations of lactobacilli can be used to modify the gut flora and thereby general metabolism and behavior not only in fishes and rodents, but also in humans [79, 122-123]. It is also known that lactobacilli or other general natural bacterial probiotics can be used as an adjuvant treatment during anticancer therapy to maintain the gut flora and stimulate the patient's immune system [see 80]. Here, we report about the beneficial pharmacological effects of a new probiotic formula on the bacterial and fungal gut microbiota occurring after one-month treatment with three bio, natural lactobacilli species in CTXtherapy. The comprehensive and comparative analysis is three-fold: (1) normal healthy condition and chemotherapy, (2) CTX + Lactobabillus and (3) different doses of Lactobacillus. We reveal the profiles of fungi and bacteria associated with long-term exposure to chemo/radio-therapy and the different doses of Lactobacillus necessary to counteract specific aversive or inhibitory effects of one-drug anticancer therapy. In particular, a systematic analysis of the gut microbiota in groups of rats in relation with cancer disease and cyclophosphamide therapy reveals the infectious bacterial pathogens that are induced by the treatment, but also the key beneficial bacterial families maintaining the immune system and protecting effects of 3L. 
L. casei complements the growth of L. acidophilus, a producer of carbohydrate-digesting enzymes, while $L$. plantarum, a more flexible and more versatile strain, produces a battery of antimicrobial substances that will help them to survive in the gastrointestinal tract in any condition. Our results in CTX-rats might pave the way to future attempts in cancer chemotherapy for humans. Fragile health conditions and microbial infections are often associated with a weakened immune system as a main consequence of "chemo". Hence, complementary and/or alternative clinical medicine must be provided for cancer prevention and/or treatment. One major finding in our study is not that cancer can be cured by $3 \mathrm{~L}$, but that the body can be rescued from chemo by a tritherapy of lactobacilli via many beneficial effects on gut flora (mycobiome and microbiome) and energy metabolism. Body composition (gut flora and metabolism) is strongly linked to the immune system that relies on energy to break down the risk of chronic diseases. Importantly, it is very likely that $3 \mathrm{~L}$ works as additive to chemical drugs not only for cancer, but also for many various metabolic diseases because the tritherapy has significantly strong beneficial effects on many various bacteria and metabolic systems (see Fig. 6). It boots the immune system and depletes specific invading agents, such as fungal pathogens (mucor) and infectious bacteria (enterococcus). Also importantly, the panoply of bacteria that are upregulated by $3 \mathrm{~L}$ treatment (see Fig. 6) is such that our probiotic formula (L. acidophilus SD65, L. casei SD07 and L. plantarum SD02) can be easily improved to target specific metabolic systems in humans. One merit of our work in rats is that different doses of lactobacillus have differential effects on the gut flora (see Fig. 6). So, further research should be undertaken to test the effects of different SD65-SD07-SD02 ratio in 3L mixtures or in combination with other mixtures of lactobacilli or beneficial bacteria (see Fig. 6). Here, we deliver a proof of research feasibility for immunization or improved immune systems in cancer. 3L may serve as a basis for the development of a large family of medicinal microbial bioproducts to be used not on chemo-animal models, but on cancer patients subject to cyclophosphamide therapy. 

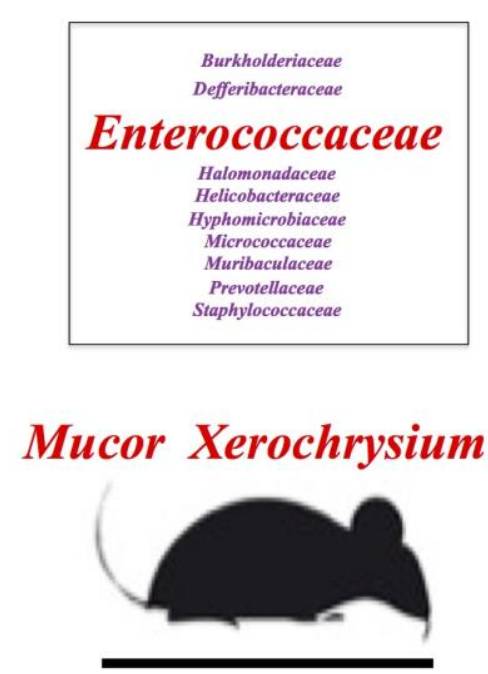

CTX
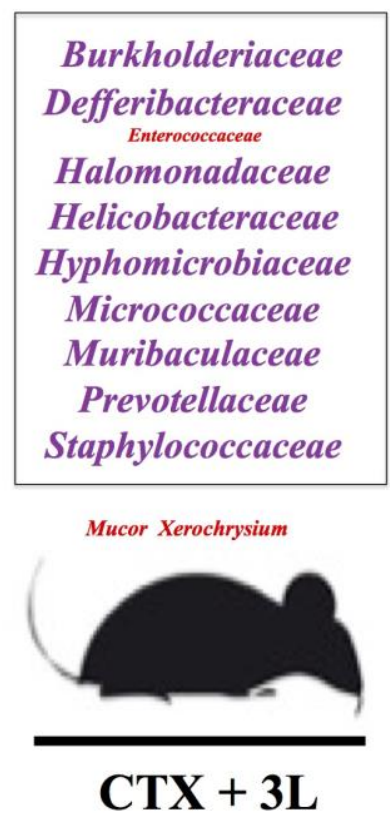

Figure 6 Regulation of gut microbiome by adding 3L to cyclophosphamide (CTX) chemotherapy in rats. Mucor, Xerochrysium, and Enterocococcacea are down-regulated by addition of 3L-Lactobacillus tritherapy to CTX treatment. A multitude of beneficial bacterial families (Burkholderiaceae, Defferibacteraceae, Halomonadaceae, Helicobacteraceae, Hyphomicrobiaceae, Muribaculaceae, Prevotellaceae and Staphylococcaceae) corresponding to various multiple metabolic pathways (see Fig. 5) are up-regulated by $3 \mathrm{~L}$.

3L (Lactobacillus bioproduct): L. acidophilus SD65, L. casei SD07, L. plantarum SD02. L: 3L-low dose $(1.25 \mathrm{mg} / \mathrm{kg}), \mathrm{M}: 3 \mathrm{~L}-$ middle dose $(2.50 \mathrm{mg} / \mathrm{kg}), \mathrm{H}: 3 \mathrm{~L}-\mathrm{high}$ dose $(5.0 \mathrm{mg} / \mathrm{kg})$.

\section{Conclusion}

The specific benefit of the formulated $3 \mathrm{~L}$ probiotic is a strong relevant action on the gut microbiome in chemotherapy conditions. While CTX and anticancer drugs have multiple potential adverse effects, which is also documented here, adding $3 \mathrm{~L}$ in anticancer therapy is proven to be beneficial to maintain gut flora and thereby health conditions. Fungal-bacterial interactions control health and disease. 3L maintains fungi and bacteria linked to health. Results on bacteria are limited to metabolic pathways, which seems to be the way $3 \mathrm{~L}$ deals with chemotherapy. The results in rats are such that the efficiency, frequency and reliability of high- 
dose retain attention over one month treatment (five CTX-chemotherapy sessions). 3L strongly preserves health conditions, maintains gut microbiota to normal conditions and strongly reinforces the host general immune defense. This is not negligible in our permanent search for tools to improve the quality of life in cancer patients.

\section{Acknowledgments}

We acknowledge Beijing Genomics Institute (BGI Co., Ltd) for Illumina MiSeq sequencing. The plan to develop a new bio-product for cancer medical industry was supported by grant supports from Shandong Province Overseas High-Level Talents Program (Taishan scholar, \#tshw20091015), Key Research and Development of Shandong Province (\#2016GGH3111), and Agricultural Science and Technology Innovation Engineering Program of Shandong Academy of Agricultural Sciences (CXGC2017A01-1).

\section{Ethics Statement}

Experiments were carried out in accordance with relevant guidelines and regulations. All experimental protocols were approved by a named institutional and licensing committee. The use of live animals (rats) in this study was approved by the Shandong Academy of Medical Sciences Ethics Committee and was licensed by Shandong Province (Governmental license SYXK (LU) 20170003).

\section{Data availability}

Our 16S and ITS sequencing data in CTX-rat model are updated at NCBI (SubmissionID: SUB9725559; BioProject ID: PRJNA754332), e.g. by the linkage of one or more BioSamples (SAMN20769197SAMN20769206; Accession Numbers: SRX11740945-SRX11740959). The locus_tag prefixes for each linked BioSample are included in the locustagprefix.txt file that can accessed from BioProject ID PRJNA754332 in the submission portal: https://submit.ncbi.nlm.nih.gov/subs/bioproject/SUB9725559/overview https://submit.ncbi.nlm.nih.gov/subs/bioproject (released January the first, 2022) 


\section{REFERENCES}

1. Hanahan, D.; Weinberg, R.A. Hallmarks of cancer: the next generation. Cell 2011, 144, 646-674.

2. Salk, J.J.; Fox, E.J.; Loeb, L.A. Mutational heterogeneity in human cancers: origins and consequences. Annu. Rev. Pathol. 2010, 5, 51-75.

3. Chakravarthi, B.V.S.K.; Nepal, S.; Varambally, S. Genomic and epigenomic alterations in cancer. J. Am. Pathol. 2016, 186, 1724-1735.

4. Mroz, E.A.; Rocco, J.W. The challenges of tumor genetic diversity. Cancer 2017, 123, 917-927.

5. Takeshima, H.; Ushijima, T. Accumulation of genetic and epigenetic alterations in normal cells and cancer risk. NPJ Precis. Oncol. 2019, 3, 7.

6. Herceg, Z; Hainaut, P. Genetic and epigenetics as biomarkers for cancer detection, diagnosis and prognosis. Mol. Oncol. 2007, 1, 26-41.

7. Berg, M.; Søreide, K. Genetic and epigenetic traits as biomarkers in colorectal cancer. Int. J. Mol. Sci. 2011, 12, 9426-9439.

8. Loeb, L.A.; Loeb, K.R.; Anderson, J.P. Multiple mutations and cancer. Proc. Natl. Acad. Sci. USA 2003, $100,776-781$.

9. Vogt, A.; Schmid, S.; Heinimann, K.; Frick, H.; Herrmann, C.; Cerny, T.; Omlin, A. Multiple primary tumours: challenges and approaches, a review. ESMO Open 2017, 2, e000172.

10. Wang, L.; Shilatifard, A. UTX mutations in human cancer. Cancer Cell 2019, 35, 168-176.

11. Chatterjee, A.; Mambo, E.; Sidransky, D. Mitochondrial DNA mutations in human cancer. Oncogene 2006, 25, 4663-4674.

12. Anastasiadou, E.; Jacob, L.S.; Slack, F.J. Non-coding RNA networks in cancer. Nat. Rev. Cancer 2013, $18,5-18$.

13. Lin, S.; Gregory, R.I. MicroRNA biogenesis pathways in cancer. Nat. Rev. Cancer 2016, 15, 321-333.

14. Gallo, L.H.; Ko, J.; Donoghue, D.J. The importance of regulatory ubiquitination in cancer and metastasis. Cell Cycle 2017, 16, 634-648.

15. Ben-Aroya, S.; Levanon, E.Y. A-to-I RNA editing: an overlooked source of cancer mutations. Cancer Cell 2018, 33, P789-790.

16. Shuai, S. et al. The U1 spliceosomal RNA is recurrently mutated in multiple cancers. Nature 2019, 574, 712-716.

17. Wang, E.; Aifantis, I. RNA splicing and cancer. Trends Cancer 2020, 6, P631-644.

18. Wilkinson, S. et al. A case report of multiple primary prostate tumors with differential drug sensitivity. Nat. Commun. 2020, 11, 837.

19. Vaklavas, C.; Blume, S.W.; Grizzle, W.E. Translational dysregulation in cancer: molecular insights and potential clinical applications in biomarker development. Front. Oncol. 2017, 7, 158.

20. Picimbon, J.F. A new view of genetic mutations. Australas. Med. J. 2017, 10, 715-718. 
21. Picimbon, J.F. "Evolution of protein physical structures in insect chemosensory systems", in Olfactory Concepts of Insect Control-Alternative to Insecticides, Vol. 2, ed. J.F. Picimbon (Springer Nature Switzerland AG), 2019, 231-263.

22. Yue, S.; Picimbon, J.F. Lactobacillus treatment on RNA editing cancer. Curr. Gene Ther. 2022, submitted.

23. Charmsaz, S.; Prencipe, M.; Kiely, M.; Pidgeon, G.P.; Collins, D.M. Innovative technologies changing cancer treatment. Cancers (Basel) 2018, 10, 208.

24. Pucci, C.; Martinelli, C.; Ciofani, G. Innovative approaches for cancer treatment: current perspectives and new challenges. Ecancermedicalscience 2019, 13, 961.

25. Waldman, A.D.; Fritz, J.M.; Lenardo, M.J. A guide to cancer immunotherapy: from T cell basic science to clinical practice. Nat. Rev. Immunol. 2020, 20, 651-668.

26. Gonzales, H.; Hagerling, C.; Werb, Z. Roles of the immune system in cancer: from tumor initiation to metastatic progression. Genes Dev. 2018, 32, 1267-1284.

27. Kaur, P.; Asea, A. Radiation-induced effects and the immune system in cancer. Front. Oncol. 2012, http://doi.org/10.3389/fonc.2012.00191.

28. Carvalho, H.A.; Villar, R.C. Radiotherapy and immune response: the systemic effects of a local treatment. Clinics 2018, 73, e557s.

29. Alfonso, J.C.L.; Papaxenopoulou, L.A.; Mascheroni, P.; Meyer-Hermann, M.; Hatzikirou, H. On the immunological consequences of conventionally fractionated radiotherapy. Science 2020, 23, 100897.

30. Lumniczky, K.; Candélias, S.M.; Gaipl, U.S.; Frey, B. Editorial: radiation and the immune system: current knowledge and future perspectives. Front. Immunol. 2018 , https://doi.org/10.3389/fimmu.2017.01933.

31. Price, J.G.; Idoyaga, J.; Salmon, H.; Hogstad, B.; Bigarella, C.L.; Ghaffari, S.; Leboeuf, M.; Merad, M. CDKN1A regulates Langerhans cell survival and promotes Treg cell generation upon exposure to ionizing irradiation. Nat. Immunol. 2015, 16, 1060-1068.

32. Scheithauer, K.; Belka, C.; Lauber, K.; Gaipl, U.S. Immunological aspects of radiotherapy. Radiat. Oncol. 2014, 9, 185.

33. Najafi, M.; Shirazi, A.; Motevaseli, E.; Geraily, Gh.; Norouzi, F.; Heidari, M.; Rezapoor, S. The melatonin immunomodulatory actions in radiotherapy. Biophys. Rev. 2017, 9, 139-148.

34. Eastham, L.L.; Howard, C.M.; Balachadran, P.; Pasco, D.S.; Claudio, P.P. Eating green: shining light on the use of dietary phytochemicals as a modern approach in the prevention and treatment of head and neck cancers. Curr. Top. Med. Chem. 2018, 18, 182-191.

35. Vivarelli, S.; Salemi, R.; Candido, S.; Falzone, L.; Santagati, M.; Stefania, S.; Torino, F.; Banna, G.L.; Tonini, G.; Libra, M. Gut microbiota and cancer: from pathogenesis to therapy. Cancers (basel) 2019, 11, 38.

36. Cheng, W.Y.; Wu, C.Y.; Yu, J. The role of gut microbiota in cancer treatment: friend or foe? Gut $\mathbf{2 0 2 0}$, 69, 1897-1876.

37. Davis, L.S. Flora-ishing guts assist cancer immunotherapies. Science Immunol. 2018, 3, eaat0813.

38. Routy, B. et al. Gut microbiome influences efficacy of PD-1-based immunotherapy against epithelial tumors. Science 2018, 359, 91-97. 
39. Li, Y. et al. Gut microbiota dependent anti-tumor immunity restricts melanoma growth in Rnf5 ${ }^{-/-}$mice. Nat. Commun. 2019, 10, 1492.

40. Ma, W.; Mao, Q.; Xia, W.; Dong, G.; Yu, C.; Jiang, F. Gut microbiota shapes the efficiency of cancer therapy. Front. Microbiol. 2019, https://doi.org/10.3389/fmicb.2019.01050.

41. Dehghani, N.; Tafvizi, F.; Jafari, P. Cell cycle arrest and anti-cancer potential of probiotic Lactobacillus rhamnosus against HT-29 cancer cells. Bioimpacts 2021, 11, 245-252.

42. Chen, L.; Li, H.; Chen, Y.; Yang, Y. Lactobacillus rhamnosus GG reduced mortality of septic mice by modulating gut microbiota composition and metabolic profiles. Nutrition 2020, 78, 110863.

43. Fong, W.; Li, Q.; Yu, J. Gut microbiota modulation: a novel strategy for prevention and treatment of colorectal cancer. Oncogene 2020, 39, 4925-4943.

44. Yue, S.; Chinnapandi, B.; Ge, H.; Zou, X.; Chen, X.; Wang, C.; Hu, W.; Picimbon, J.F. A Lactobacillus cocktail changes gut flora and reduces cholesterolemia and weight gain in hyperlipidemia mice. SOJ Microbiol. Infect. Dis. 2014, 2, 1-16.

45. Yue, S.; Liu, X.; Fu, J. Lactobacillus tritherapy for cholesterol and heart diseases. RR J. Microbiol. Biotechnol. 2016, 5, 23-26.

46. Tian, H.; Lyu, Y.; Yang, Y.G.; Hu, Z. Humanized rodent models for cancer research. Front. Oncol. 2020, https://doi.org/10,3389/fonc.2020.01696.

47. Czpirer, C. "Cancer research in rat models", in Rat Genomics. Methods in Molecular Biology (Methods and Protocols), Vol. 597, ed. I. Anegon (Humana Press), 2010, 445-458.

48. Zhang, Z.; Yue, S.; Chen, L.; Teng, S.; Sun, P.; Wang, Y.; Li, J. Effect of Lactobacillus preparation on immunosuppression induced by cyclophosphamide in rats. Food Drug 2020, 22, 103-107.

49. Yue, S.; Li, Z.; Hu, F.; Picimbon, J.F. Curing piglets from diarrhea and preparation of a healthy microbiome with Bacillus treatment for industrial animal breeding. Sci. Rep. 2020, 10, 19476.

50. Human Genome Assembly: The Genome Sequencing Consortium. Initial sequencing and analysis of the human genome. Nature 2001, 409, 860-921.

51. Qin, J. et al. A human gut microbial gene catalogue established by metagenomic sequencing. Nature 2010, 464, 59.

52. Ravi, R.K.; Walton, K.; Khosroheidari, M. "MiSeq: A next generation sequencing platform for genomic analysis", in Disease Gene Identification. Methods in Molecular Biology, Vol. 1706, ed. J. DiStefano (Humana Press), 2018, 223-232.

53. Caporaso, J.G. et al. QIIME allows analysis of high-throughput community sequencing data. Nat. Methods 2010, 7, 335-336.

54. Edgar, R.C. Search and clustering orders of magnitude faster than BLAST. Bioinformatics 2010, 26, 2460-2461.

54. Bolyen, E. et al. Reproducible, interactive, scalable and extensible microbiome data science using QIIME 2. Nat Biotechnol. 2019, 37, 852-857.

55. Blaxter, M.; Mann, J.; Chapman, T.; Thomas, F.; Whitton, C.; Floyd, R.; Abebe, E. Defining operational taxonomic units using DNA barcode data. Philos. Trans. R. Soc. Lond. B. Biol. Sci. 2005, 360, 1935-1943. 
56. Cameron, E.S.; Schmidt, P.J.; Tremblay, B.J.; Emelko, M.B.; Müller, K.M. To rarefy or not to rarefy: enhancing microbial community analysis through next-generation sequencing. BioRxiv 2020, DOI:10.1101/2020.09.09.290049.

57. Blaxter, M.; Mann, J.; Chapman, T.; Thomas, F.; Whitton, C.; Floyd, R.; Abebe, E. Defining operational taxonomic units using DNA barcode data. Philos. Trans. R. Soc. Lond. B. Biol. Sci. 2005, 360, 1935-1943.

58. Gotelli, N.J.; Colwell, R.K. Quantifying biodiversity: procedures and pitfalls in the measurement and comparison of species richness. Ecol. Lett. 2001, 4, 379-391.

59. Oksanen, J.; Kindt, R.; Legendre, P.; O’Hara, B.; Steven, M.H.H.; Oksanen, M.J. The vegan package. Community Ecol. package 2007, 10, 631-637.

60. Izsák, J.; Pavoine, S. Links between the species abundance distribution and the shape of the corresponding rank abndance curve. Ecol. Indic. 2012, 14, 1-6.

61. White, J.R.; Nagarajan, N.; Pop, M. Statistical methods for detecting differentially abundant features in clinical metagenomic samples. PLoS Comput. Biol. 2009, 5, e1000352.

62. Segata, N.; Izard, J.; Waldron, L.; Gevers, D.; Miropolsky, L.; Garrett, W.S.; Huttenhower, C. Metagenomic biomarker discovery and explanation. Genome Biol. 2011, 12, R60.

63. Price, M.N.; Dehal, P.S.; Arkin, A.P. FastTree: computing large minimum-evolution trees with profiles instead of a distance matrix. Mol. Biol. Evol. 2009, 26, 1641-1650.

64. Huson, D.H. et al. Integrative analysis of environmental sequences using MEGAN4. Genome Res. 2011, $21,1552-1560$.

65. Asnicar, F.; Weingart, G.; Tickle, T.L.; Huttenhower, C.; Segata, N. Compact graphical representation of phylogenetic data and metadata with GraPhlAn. Peer J. 2015, 3, e1029.

66. Ondov, B.D.; Bergman, N.H.; Phillippy, A.M. Interactive metagenomic visualization in a Web browser. BMC Inform. 2011, 12, 385.

67. Su, L.; Zhu, H.; Guo, Y.; Du, X.; Jianguo, G.; Zhang, L.; Qin, C. Lecanicillium coprophilum (Cordypitaceae, Hypocreales), a new species of fungus from the feces of Marmota monax in China. Phytotaxa 2019, 387, 55-62.

68. Jermy, A. Ustilago gives plants a Pep talk. Nat. Rev. Microbiol. 2012, 10, 444-445.

69. Verma, R.K.; Pandro, V.; Raj, D.; Patel, D.; Asaiya, A.J.K. Diversity of macro-fungi in Central IndiaXX: Phallus atrovolvatus and Phallus merulinus. Van Sangyan 2019, 6, 1-8.

70. Rota, A.; Calicchio, E.; Nardoni, S.; Fratini, F.; Ebani, V.V.; Sgorbini, M.; Panzani, D.; Camillo, F.; Manciati, F. Presence and distribution of fungi and bacteria in the reproductive tract of healthy stallions. Theriogenology 2011, 76, 464-470.

71. Su, L.; Zhu, H.; Guo, Y.; Du, X.; Jianguo, G.; Zhang, L.; Qin, C. Lecanicillium coprophilum (Cordypitaceae, Hypocreales), a new species of fungus from the feces of Marmota monax in China. Phytotaxa 2019, 387, 55-62.

72. McGhie, T.A.; Huber, T.W.; Kassis, C.E.; Jinadatha, C. Ustilago species as a cause of central line-related blood stream infection. Am. J. Med. Sci. 2013, 345, 254-255.

73. Huseyin, C.E.; O’Toole, P.W.; Cotter, P.D.; Scanlan, P.D. Forgotten fungi-the gut mycobiome in human health and disease. FEMS Microbiol. Rev. 2017, 41, 479-511. 
74. Sam, Q.H.; Chang, M.W.; Chai, L.Y. The fungal mycobiome and its interaction with gut bacteria in the host. Int. J. Mol. Sci. 2017, 18, 330.

75. Li, J.; Chen, D.; Yu, B.; He, J.; Zheng, P.; Mao, X.; Yu, J.; Luo, J.; Tian, G.; Huang, Z.;Luo, Y. Fungi in gastrointestinal tracts of human and mice: from community to functions. Microb. Ecol. 2018, 75, 821-829.

76. Caspi, R.; Billington, R.; Fulcher, C.A.; Keseler, I.M.; Kothari, A.; Krummenacker, M.; Latendresse, M.; Midford, P.E.; Ong, Q.; Ong, W.K.; Paley, S.; Subhraveti, P.; Karp, P.D. The MetaCyc database of metabolic pathways and enzymes. Nucleic Acids Res. 2018, 46, D633-D639.

77. Hove, H.; Norgaard, H.; Mortensen, P.B. Lactic acid bacteria and the human gastrointestinal tract. Eur. J. Clin. Nutr. 1999, 53, 339-350.

78. Fooks, L.J.; Gibson, G.R. Probiotics as modulators of the gut flora. Br. J. Nutr. 2002, 88, S39-49.

79. Walter, J. Ecological role of Lactobacilli in the gastrointestinal tract: implications for fundamental and biomedical research. Appl. Environ. Microbiol. 2008, 74, 4985-4996.

80. Hemarajata, P.; Versalovic, J. Effects of probiotics on gut microbiota: mechanisms of intestinal immunomodulation and neuromodulation. Therap. Adv. Gastroenterol. 2013, 26, 39-51.

81. Panebianco, C.; Latiano, T.; Pazienza, V. Microbiota manipulation by probiotics administration as emerging tool in cancer prevention and therapy. Front. Oncol. 2020, https://doi.org/10.3389/fonc.2020.00679.

82. Lu, K.; Dong, S.; Wu, X.; Jin, R.; Chen, H. Probiotics in cancer. Front. Oncol. 2021, https://doi.org/10.3389/fonc.2021.638148.

83. Yu, A.Q.; Li, L. The potential role of probiotics in cancer prevention and treatment. Nutr. Cancer 2016, $68,535-544$.

84. Bedada, T.L.; Feto, T.K.; Awoke, K.S.; Garedew, A.D.; Yifat, F.T.; Birri, D.J. Probiotics for cancer alternative prevention and treatment. Biomed. Pharmacother. 2020, 129, 110409.

85. Aykut, B. et al. The fungal mycobiome promotes pancreatic oncogenesis via activation of MBL. Nature 2019, 574, 267-267.

86. Walter, J. Ecological role of Lactobacilli in the gastrointestinal tract: implications for fundamental and biomedical research. Appl. Environ. Microbiol. 2008, 74, 4985-4996.

87. Korula, A.; Abraham, A.; Abubacker, F.N.; Viswabandya, A.; Lakshmi, K.M.; Abraham, O.C.; Rupali, P.; Varghese, G.M.; Michael, J.S.; Srivastava, A.; Mathews, V.; George, B. Invasive fungal infection following chemotherapy for acute myeloid leukaemia-Experience from a developing country. Mycoses 2017, 60, 686-691.

88. Iida, N.; Mizukoshi, E.; Yamashita, T.; Terashima, T.; Arai, K.; Seishima, J.; Kaneko, S. Overuse of antianaerobic drug is associated with poor postchemotherapy prognosis of patients with hepatocellular carcinoma. Int. J. Cancer 2019, 145, 2701-2711.

89. Wainwright, M. Some highlights in the history of fungi in medicine - a personal journey. Fungal Biol. Rev. 2008, 22, 97-102.

90. Nishad, J.H.; Singh, A.; Gautam, V.S.; Kumar, D.; Kumar, J.; Kharwar, R.N. "Endophytic fungi: a cryptic fountainhead for biodiversity, functional metabolites, host stress tolerance, and myco-mediated nanoparticles (Nps) synthesis", in Endophytes and Secondary Metabolites, ed. S. Jha (References Series in Phytochemistry, Springer Cham), 2018, 1-29. 
91. Lombard, L.; van der Merwe, N.A.; Groenewald, J.Z.; Crous, P.W. Generic concepts in Nectriaceae. Stud. Mycol. 2015, 80, 189-245.

92. Fraiser, L.H.; Kanekal, S.; Kehrer, J.P. Cyclophosphamide toxicity. Characterising and avoiding the problem. Drugs 1991, 42, 781-795.

93. Nicolás, F.E.; Murcia, L.; Navarro, E.; Navarro-Mendoza, M.I.; Pérez-Arques, C.; Garre, V. Mucorales species and macrophages. J. Fungi (Basel) 2020, 6, 94.

94. Rosenblum, E.B.; Voyles, J.; Poorten, T.J.; Stajich, J.E. The deadly chytrid fungus: a story of an emerging pathogen. PLoS Pathog. 2010, 6, e1000550.

95. Witherden, E.A.; Shoale, S.; Hall, R.A.; Moyes, D.L. The human mucosal mycobiome and fungal community interactions. J. Fungi 2017, 3, 56.

96. Jayasudha, R.; Das, T.; Chakravarthy, S.K.; Prashanthi, G.S.; Bhargava, A.; Tyagi, M.; Rani, P.K.; Pappuru, R.R.; Shivaji, S. Gut mycobiomes are altered in people with type 2 Diabetes Mellitus and Diabetic Retinopathy. PLOS ONE 2020, 15, e0243077.

97. Coker, O.O.; Nakatsu, G.; Dai, R.Z.; Wu, W.K.K.; Wong, S.H.; Ng, S.C.; Chan, F.K.L.; Sung, J.J.Y.; Yu, J. Enteric fungal microbiota dysbiosis and ecological alterations in colorectal cancer. Gut 2019, 68, 654-662.

98. van Tilburg Bernardes, E. et al. Intestinal fungi are causally implicated in microbiome assembly and immune development in mice. Nat. Commun. 2020, 11, 2577.

99. Ghannoum, M.A.; Jurevic, R.J.; Mukherjee, P.K.; Cui, F.; Sikaroodi, M.; Naqvi, A.; Gillevet, P.M. Characterization of the oral fungal microbiome (mycobiome) in healthy individuals. PLoS Pathog. 2010, 6, e1000713.

100. Jørgensen, M.R.; Kragelund, C.; Jensen, P.Ø.; Keller, M.K.; Twetman, S. Probiotic Lactobacillus reuteri has antifungal effects on oral Candida species in vitro. J. Oral Microbiol. 2017, 9, 1274582.

101. Locasale, J.W.; Cantley, L.C. Altered metabolism in cancer. BMC Biol. 2010, 8, 88.

102. Heske, CM. Beyond energy metabolism: exploiting the additional roles of NAMPT for cancer therapy. Front. Oncol. 2020, 9, 1514.

103. Desbats, M.A.; Giacomini, I.; Prayer-Galetti, T.; Montopoli, M. Metabolic plasticity in chemotherapy resistance. Front. Oncol. 2020, 10, 281.

104. Van Soom, T.; El Bakkali, S.; Gebruers, N.; Verbelen, H.; Tjalma, W.; van Breda, E. The effects of chemotherapy on energy metabolic aspects in cancer patients: a systematic review. Clin. Nutr. 2020, 39, 1863-1877.

105. Chung, Y.W.; Gwak, H.J.; Moon, S.; Rho, M.; Ryu,, J.H. Functional dynamics of bacterial species in the mouse gut microbiome revealed by metagenomic and metatranscriptomic analyses. PLoS ONE 2020, 15, e0227886.

106. Kovatcheva-Datchary, P.; Nilsson, A.; Akrami, R.; Lee, Y.S.; De Vadder, F.; Arora, T.; Hallen, A.; Martens, E.; Björck, I.; Bäckhed, F. Dietary fiber-induced improvement in glucose metabolism is associated with increased abundance of Prevotella. Cell Metabol. 2015, 22, 971-982.

107. Polansky, O.; Sekelova, Z.; Faldynova, M.; Sebkova, A.; Sisak, F.; Rychlik, I. Important metabolic pathways and biological processes expressed by chicken cecal microbiota. Appl. Environ. Microbiol. 2016, $82,1569-1576$.

108. Franke, T.; Deppenmeier, U. Physiology and central carbon metabolism of the gut bacterium Prevotella copri. Mol. Microbiol. 2018, 109, 528-540. 
109. Precup, G.; Vodnar, D.C. Gut Prevotella as a possible biomarker of diet and its eubiotic versus dysbiotic roles: a comprehensive literature review. Brit. J. Nutr. 2019, 122, 131-140.

110. Owyang, S.Y.; Luther, J.; Kao, J.Y. Helicobacter pylori: beneficial for most? Expert Rev. Gastroenterol. Hepatol. 2011, 5, 649-651.

111. Abadi, A.T.B. Helicobacter pylori: a beneficial gastric pathogen? Front. Med. (Lausanne) 2014, 1, 26.

112. Marais, M.; Mendz, G.L.; Hazell, S.L.; Mégraud, F. Metabolism and genetics of Helicobacter pylori: the genome era. Microbiol. Mol. Biol. Rev. 1999, 63, 642-674.

113. Weir, S.; Cuccherini, B.; Whitney, A.M.; Ray, M.L.; MacGregor, J.P.; Steigerwalt, A.; Daneshvar, M.I.; Weyant, R.; Wray, B.; Steele, J.; Strober, W.; Gill, V.J. Recurrent bacteremia caused by a "Flexispira"-like organism in a patient with X-linked (Bruton's) aggammaglobulinemia. J. Clin. Microbiol. 1999, 37, 24392445 .

114. Iten, A.; Graf, S.; Egger, M.; Tauber, M.; Graf, J. Helicobacter sp. flexispira bacteremia in an immunocompetent young adult. J. Clin. Microbiol. 2001, 39, 1716-1720.

115. Wei, G.; Tian, N.; Siezen, R.; Schuppan, D.; Helmerhorst, E.J. Identification of food-grade substilisins as gluten-degrading enzymes to treat celiac disease. Am. J. Physiol. Gastrointest. Liver Physiol. 2016, 311, G571-G580.

116. Moore, R.E.; Townsend, S.D. Temporal development of the infant gut microbiome. Open Biol. 2019, 9 , 190128.

117. Lu, C.; Xia, Y.; Liu, D.; Zhao, R.; Liu, H.; Xun, L. Cupriavidus necator H16 uses flavocytochrome c sulfide dehydrogenase to oxidize self-produced and added sulfide. Appl. Environ. Microbiol. 2017, 83, e01610-17.

118. Sosa, O.A. Phosphorus redox reactions as pinch hitters in microbial metabolism. Proc. Natl. Acad. Sci. USA 2018, 115, 7-8.

119. Talwar, C.; Nagar, S.; Kumar, R.; Scaria, J.; Lal, R.; Negi, R.K. Defining the environmental adaptations of genus Devosia: insights into its expansive short peptide transport system and positively selected genes. Sci. Rep. 2020, 10, 1151.

120. McMahon, R.J. Biotin in metabolism and molecular biology. Annu. Rev. Nutr. 2002, 22, 221-239.

121. Vieira de Almeida, C.; Taddei, A.; Amedei, A. The controversial role of Enterococcus faecalis in colorectal cancer. Therap. Adv. Gastroenterol. 2018, 11, 1756284818783606.

122. Falcinelli, S.; Rodiles, A.; Unniappan, S.; Picchietti, S.; Gioacchini, G.; Merrifield, D.L.; Carnevalli, O. Probiotic treatment reduces appetite and glucose level in the zebrafish model. Sci. Rep. 2016, 6, 18061.

123. Wang., M.; Chen, Y.; Wang, Y.; Li, Y.; Zhang, X.; Zheng, H.; Ma, F.; Ma, C.; Lu, B.; Xie, Z.; Liao, Q. Beneficial changes of gut microbiota and metabolism in weaned rats with Lactobacillus acidophilus NCFM and Bifidobacterium lactis Bi-07 supplementation. J. Funct. Foods 2018, 48, 252-265. 


\section{LEGEND SUPPLEMENTARY FIGURES}

Figure S1. Classification of OTUs at different taxonomic levels. Differences of OTU composition in fifteen samples corresponding to five groups of rats in relation with cancer and CTX chemotherapy + Lactobacillus. CK: normal (control healthy), IM: immune-damaged (CTX-treated, ill), L: chemotherapy + 3L-low dose, M: chemotherapy + 3L-middle dose, H: chemotherapy + 3L-high dose. CK: C101, C103, C105; IM: IM015, IM021, IM024; L: L102, L103, L104; M: M201, M202, M203; H: H105, H204, H205. The number of OTUs at a specific taxonomic level is directly displayed (color code). "Phylum", "Class", "Order", "Family", "Genus" and "Species" represent the number of OTUs that can be classified into the taxonomic level of Phylum, Class, Order, Family, Genus and Species. "Unclassified represents the number of OTUs that cannot be attributed to any known taxon (R(v3.1.1), BGI Co., Ltd).

Figure S2. Principal Component Analysis and fungal species matrix in five groups of rats related to cancer, chemo and Lactobacillus. A) Partial Least Squares Discriminant Analysis (PLS-DA) in CK-H groups. Fungal species matrix (left) and sample grouping data (right) based on Variable Importance in Projection (VIP) of each species. VIP>1, the larger the value, the greater contribution of the species to the differences between groups. Mucor greatly contributes to the differences between IM (immune-attacked, ill, CTX, chemo) group and the other groups (CK: control healthy conditions and L-H: Lactobacillus-treated). Lactobacillus tritherapy: L. acidophilus SD65, L. casei SD07, L. plantarum SD02 (3L). L: 3L-low dose (1.25 mg/kg), M: 3L-middle dose $(2.50 \mathrm{mg} / \mathrm{kg}), \mathrm{H}: 3 \mathrm{~L}-\mathrm{high}$ dose $(5.0 \mathrm{mg} / \mathrm{kg})$. Each point or dot represents a sample, dots of the same color belong to the same group, and same groups (three points or dots) were marked by ovals (right). The closer the distance of same groups, and the farther the distance of different groups, the better of the classification model. On PLS-DA classification model for rats related to cancer and chemotherapy, H-dose and CK groups fall very close to each other, with IM as the far point (R(v3.1.1), BGI Co., Ltd). B) Orthogonal projections to Latent Structures Discriminant Analysis (OPLS-DA). Fusarium and sarocladium strongly contribute to the differences between CK-H groups and IM (see *). Talaromyces associates with IM conditions, but could be cured by 3L-low dose (see *). L-dose of 3L bioproduct is less efficient on mucor $($ see $*)$. 
Figure S3. Rank abundance curve (OTU richness) of each group of rats related to cancer and chemotherapy. The abscissa represents the OTU number, and the ordinate represents the OTU richness (converted by Log2 algorithm) in one group. Each line represents the OTU abundance distribution of a sample or group (CK-H). The length of the line on the abscissa reflects the number of OTUs in the sample or group, which represents the richness of the microbial community. For microbial community samples, the curve can visually indicate the number of highly abundant and rare OTUs in each community. The OTUs of each sample are arranged in descending order according to their abundance. The rank abundance curves of CK and Lactobacillus display similar relative microbial species abundance and diversity, an essential component of healthy microbiome. 3L (Lactobacillus): L. acidophilus SD65, L. casei SD07, L. plantarum SD02. L: 3L-low dose (1.25 mg/kg), M: 3L-middle dose (2.50 mg/kg), H: 3L-high dose (5.0 mg/kg). IM: Immune-attacked (CTX treatment).

Figure S4. Unweighted UniFrac Nonmetric MultiDimensional Scaling analysis of cancer rat groups with 3L. Each point represents a sample, and the points of different colors belong to different samples (groups/ CK: control healthy conditions; IM: immune-attacked, ill, CTX treatment; L: 3L-low dose; M: 3L-middle dose; H: 3L-high dose). The closer the distance between the two points, the higher the similarity of the microbial community structure between the two samples. A) Bray-Curtis index (common, ellipse and hull). B) Jaccard index (common, ellipse and hull). A-B) The distance between the two samples or groups or samples is very close, the similarity of the microbial community structure between the two samples or groups (in A: CK and 3L; in B: CK and $\mathrm{H})$ is very high ( $\mathrm{R}(\mathrm{v} 3.1 .1)$, BGI Co., Ltd).

Figure S5. Beta-diversity analysis of five rat groups in relation with cancer, chemotherapy and Lactobacillus.. Each point represents a sample, and the points of different colors belong to different samples (five groups/ CK: control healthy conditions; IM: immune-attacked, ill, CTX-treated; L: 3L-low dose; M: 3L-middle dose; H: 3L-high dose). The closer the distance between the two points or dots, the higher the similarity of the microbial community structure between the two samples. The percentage of the parentheses in the coordinates (X-axis, $1^{\text {st }}$ principal component, Axis 1 ; Y-axis, $2^{\text {nd }}$ principal component, Axis 2 ) represents the 
proportion of the differences in the original data that can be explained by the corresponding principal component. A) Principal Coordinates Analysis (PCoA) derived from unweighted and weighted UniFrac and Bray-Curtis index (common, ellipse and hull). B) Principal Coordinates Analysis (PCoA) derived from unweighted and weighted UniFrac and Jaccard index (common, ellipse and hull). A-B) The distance between the two groups is very close, the similarity of the microbial community structure between the two groups (in A: CK and 3L; in B: CK and H) is very high (R(v3.1.1), BGI Co., Ltd).

Figure S6. Unweighted pair-group method with arithmetic means (UPGMA) clustering analysis of cancer rat groups with 3L (Lactobacillus tritherapy). Fifteen samples/CK: C101, C103, C105; IM: IM015, IM021, IM024; L: L102, L103, L104; M: M201, M202, M203; H: H105, H204, H205. Five groups/CK: control healthy conditions; IM: immune-attacked, ill, chemo, cyclophosphamide CTX-treated; L: 3L-low dose; M: 3L-middle dose; H: 3L-high dose. The samples are clustered according to the similarity between each other, and the shorter the branch length between the samples, the more similar the two samples. A) UPGMA cluster based on Bray-Curtis dissimilarity index. B) UPGMA cluster based on Jaccard similarity index. In A and B, CK and Lactobacillus $(\mathrm{H})$ samples clustered together. The branch length is very short between CK and $\mathrm{H}$. CK and H-dose samples are very similar (see *) (R(v3.1.1), BGI Co., Ltd).

Figure S7. Specaccum species accumulation curve of fifteen samples from cancer rat groups with 3L therapy. The abscissa represents the sample number, the ordinate represents the number of microbial species detected, and the blue shade reflects the confidence interval of the curve. The results based on fifteen samples reflect the increasing rate of new detected species along with the increase of sample number. Except for three dots, the curve shows a linear rise in the form and tends to be flat. The linear increase and the flat curve indicate that the sample number is sufficient to reflect the community richness (R(v3.1.1), BGI Co., Ltd).

Figure S8 Alpha-diversity analysis of five rat groups in relation with cancer, chemotherapy and Lactobacillus. Different histograms based on Chao1, Simpson, Shannon, Pielou_e, Observed_species and Goods_coverage ( $\mathrm{p}=0.16-0.76$; R(v3.1.1), BGI Co., Ltd) show similar alpha-diversity between CK and H groups (see *). CK: 
control healthy conditions; IM: immune-attacked, ill, cyclophosphamide CTX-treated; L: 3L-low dose; M: 3L-middle dose; H: 3L-high dose.

Figure S9. Fungal taxa distribution in rat groups in relation with cancer and chemotherapy at phylum, order, class, family and genus level. A) Phylum-level. B) Class-level, C) Order-level, D) Family-level and E) Genus-level. Fifteen samples/CK: C101, C103, C105; IM: IM015, IM021, IM024; L: L102, L103, L104; M: M201, M202, M203; H: H105, H204, H205. Five groups/CK: control healthy conditions; IM: immuneattacked, ill, chemo, cyclophosphamide CTX-treated; L: 3L-low dose; M: 3L-middle dose; H: 3L-high dose. The relative abundance of each fungal taxa in a specific group or sample is directly displayed (color code). At phylum-to-genus level, top 20 most abundant fungal taxa in rat fecal samples were used to draw the histogram. The curve shows down-regulation of the rat mycobiome after chemotherapy, but up-regulation of the mycobiome during high- (and middle-) dose 3L therapy (A-E, R(v3.1.1), BGI Co., Ltd).

Figure S10. MetagenomeSeq of enriched microbes in control healthy conditions versus immune-damaged. Comparative analysis of fungal abundance between metagenomes from control (CK) and IM groups in rats. MetagenomeSeq detects the highest number of taxa in complete fungal dataset (R(v3.1.1), BGI Co., Ltd). Trichosporonales (Basidiomycota) are differentially abundant fungi between two groups (CK and IM) of multiple samples, as found for 3L (high- and middle-dose) Lactobacillus therapy (see Fig. S9).

Figure S11. LefSe analysis, linear discriminant analysis with nonparametric Kruskal-Wallis and Wilcoxon rank sum test. Identification of key fungal biomarkers (i.e. key mycobiome member) in different samples or groups of rats related to cancer and chemotherapy. LefSe only shows statistically significant differences between sample groups (identified as “class”). A) Relative abundance distribution of classification units with significant differences in different groups. CK: control healthy conditions; IM: immune-attacked, ill, chemo, cyclophosphamide CTX-treated; L: 3L-low dose; M: 3L-middle dose; H: 3L-high dose. LefSe identifies significantly different classification units (Fungi, Ascomycota, Sordariomycetes, Hypocreales, Nectriaceae, Fusarium between CK-H and IM-L-M classes) in solid and dashed lines, respectively. In each unit packet, the relative abundance of the mean and median reflect the size of the differences between groups (see 
Fusarium*). B) Intergroup differential classification unit display chart based on classification tree (1e-04, 1e05). Hierarchical relationships of all taxa from phylum to genus (i.e. from the inner circle to the outer circle) in the sample set. The node size corresponds to the average relative abundance of the taxon. The letter a identifies the name of the classification unit where there is a significant difference between groups. Fusarium has a higher abundance in the sample or group (CK) represented by green (see *, R(v3.1.1), BGI Co., Ltd).

Figure S12. Interaction network according to group and fungal microbial species composition. A) Network according to groups of rats in relation with cancer, chemotherapy and 3L Lactobacillus bioproduct treatment. CK: control healthy conditions; IM: immune-attacked, ill, chemo, cyclophosphamide CTX-treated; L: 3Llow dose; M: 3L-middle dose; H: 3L-high dose. Network of CK samples (in yellow) interact with H network (in green). IM samples (in blue) fall more distantly and do not build a network. B) Network according to phylum. Network of CK-H samples coincides with Ascomycota network (see *, R(v3.1.1), BGI Co., Ltd).

Figure S13. Histogram of metabolic reactions (MetaCyc base pathways) measured in five groups of rats related to cancer and chemotherapy (+3L). A) Relative abundance of reactions. B) Reaction counts. 